International Journal of Applied Mathematics

Volume 33 No. $1 \quad 2020,75-98$

ISSN: 1311-1728 (printed version); ISSN: 1314-8060 (on-line version)

doi: http://dx.doi.org/10.12732/ijam.v33i1.7

\title{
EXTENDED FRACTIONAL INTEGRALS, PRODUCTS AND RATIOS OF MATRICES AND STATISTICAL DISTRIBUTIONS
}

\author{
A.M. Mathai \\ Emeritus Professor of Mathematics and Statistics \\ McGill University, CANADA
}

\begin{abstract}
For two functions, Mellin convolutions of products and ratios are well-known in the literature. But these for three or more functions is not discussed extensively in the literature. The corresponding matrix transform and M-convolutions are rarely considered in the literature. In this paper, a detailed examination of $\mathrm{M}$-convolutions of products and ratios involving three functions is carried out, pointing out the relevance of the results in different areas such as fractional calculus, especially fractional integrals, and statistical distribution theory.
\end{abstract}

AMS Subject Classification: 26A33, 44A05, 44A35, 62EXX, 62H10, 15B52 Key Words: fractional integrals; statistical distributions; matrix-variate functions; Mellin convolutions of products and ratios; M-transforms

\section{Introduction}

In this paper, real scalar mathematical as well as random variables will be denoted by small letters $x, y, \ldots$ and the corresponding complex scalar variables will be denoted by $\tilde{x}, \tilde{y}, \ldots$ with a tilde. Real vector/matrix variables, whether mathematical or random, will be denoted by capital letters $X, Y, \ldots$ and the corresponding variables in the complex domain will be denoted with a tilde such as $\tilde{X}, \tilde{Y}, \ldots$ Constant scalars will be denoted by $a, b, \ldots$ and constant matrices will be denoted by $A, B, \ldots \ldots$. Let $x$ and $y$ be real scalar variables with $\mathrm{d} x$ and $\mathrm{d} y$ denoting their differentials. Then the wedge product of differentials

Received: August 23, 2019

(c) 2020 Academic Publications 
is defined as $\mathrm{d} x \wedge \mathrm{d} y=-\mathrm{d} y \wedge \mathrm{d} x$ so that $\mathrm{d} x \wedge \mathrm{d} x=0, \mathrm{~d} y \wedge \mathrm{d} y=0$. If $X$ is a $p \times 1$ vector with $X^{\prime}=\left(x_{1}, \ldots, x_{p}\right)$, a prime denoting the transpose, then $\mathrm{d} X=\mathrm{d} x_{1} \wedge \ldots \wedge \mathrm{d} x_{p}=\mathrm{d} X^{\prime}$ whenever $x_{j}$ 's are real distinct or functionally independent. If $X=\left(x_{i j}\right)$ is a $m \times n$ real matrix with all $x_{i j}$ 's distinct then $\mathrm{d} X=\wedge_{i=1}^{m} \wedge_{j=1}^{n} \mathrm{~d} x_{i j}$. If $X=X^{\prime}$ is $p \times p$ then $\mathrm{d} X=\wedge_{i \geq j} \mathrm{~d} x_{i j}=\wedge_{i \leq j} \mathrm{~d} x_{i j}$ or there are only $p(p+1) / 2$ differentials in $\mathrm{d} X$. If $\tilde{X}$ is $m \times n$ and in the complex domain then $\tilde{X}=X_{1}+i X_{2}, i=\sqrt{(-1)}, X_{1}, X_{2}$ real. Then $\mathrm{d} \tilde{X}$ is defined as $\mathrm{d} \tilde{X}=\mathrm{d} X_{1} \wedge \mathrm{d} X_{2}$. If $X$ is $p \times p$ real positive definite then it is written as $X=X^{\prime}>O$. If $\tilde{X}$ is $p \times p$ and in the complex domain then $\tilde{X}^{*}$ will denote its conjugate transpose. If $\tilde{X}=\tilde{X}^{*}$ then $\tilde{X}$ is Hermitian and if $\tilde{X}=\tilde{X}^{*}>O$ then $\tilde{X}$ is Hermitian positive definite. Let $f(Z)$ be a real-valued scalar function of $Z$ where $Z$ may be real scalar, vector, matrix or complex scalar, vector, matrix, then if $f(Z) \geq 0$ for all $Z$ and $\int_{Z} f(Z) \mathrm{d} Z=1$ then $f(Z)$ is called a density function of $Z$. The integral $\int_{A<X<B} f(X) \mathrm{d} X=\int_{A}^{B} f(X) \mathrm{d} X$ will mean $A, B, X$ are $p \times p$ real positive definite, where $A$ and $B$ are constant matrices, $f(X)$ is a real-valued scalar function of $X$ and the integral is taken over $A>O, B>O, X>O, X-A>O, B-X>O$. The corresponding notation will be used in the complex case.

For a $p \times p$ matrix $X, \operatorname{tr}(X)$ means the trace of $X,|X|=\operatorname{det}(X)$ means the determinant of $X$. When $\tilde{X}$ is in the complex domain then $|\operatorname{det}(\tilde{X})|$ means the absolute value of the determinant of $\tilde{X}$. In general $\operatorname{det}(\tilde{X})=a+i b$ where $a$ and $b$ are real scalars and $i=\sqrt{(-1)}$. Then $\operatorname{det}\left(\tilde{X}^{*}\right)=a-i b, \operatorname{det}(\tilde{X}) \operatorname{det}\left(\tilde{X}^{*}\right)=(a+$ $i b)(a-i b)=a^{2}+b^{2}=\operatorname{det}\left(\tilde{X} \tilde{X}^{*}\right)$ and $|\operatorname{det}(\tilde{X})|=+\sqrt{\left(a^{2}+b^{2}\right)}$. A real-valued scalar function $f(X)$ of $X$ is said to be a symmetric function or functionally symmetric when $f(A B)=f(B A)$ where $A B$ need not be equal to $B A$. When $f(X)$ is symmetric and if the $p \times p$ real matrix $X=X^{\prime}$, then $f(X)=f\left(Q Q^{\prime} X\right)=$ $f\left(Q^{\prime} X Q\right)=f(D), D=\operatorname{diag}\left(\lambda_{1}, \ldots, \lambda_{p}\right)$ where $\lambda_{1}, \ldots, \lambda_{p}$ are the eigenvalues of $X$ and $Q$ is orthonormal or $Q Q^{\prime}=I, Q^{\prime} Q=I, Q^{\prime} X Q=D$. When $\tilde{X}$ is Hermitian then a real-valued scalar function $\tilde{f}(\tilde{X})=\tilde{f}(D), D=\operatorname{diag}\left(\lambda_{1}, \ldots, \lambda_{p}\right)$, where $\lambda_{1}, \ldots, \lambda_{p}$ are real and the eigenvalues of $\tilde{X}=\tilde{X}^{*}$.

For $x>0$ real scalar, the Mellin transform of a function $f(x)$, with Mellin parameter $s$, is defined and denoted as

$$
M_{f}(s)=\int_{0}^{\infty} x^{s-1} f(x) \mathrm{d} x
$$

whenever the integral is convergent. Corresponding matrix transform or Mtransform of $f(X)$ for the $p \times p$ real positive definite matrix $X>O$, with parameter $\rho$, is defined and denoted as

$$
M_{f}(\rho)=\int_{X>O}|X|^{\rho-\frac{p+1}{2}} f(X) \mathrm{d} X, \Re(\rho)>\frac{p-1}{2}
$$


whenever the integral is convergent, where $\Re(\cdot)$ denotes the real part of $(\cdot)$. Let $x_{1}>0, x_{2}>0$ be real scalar variables, mathematical or random. Let $u_{12}=x_{1} x_{2}$ the product. Then the transformation $\left(x_{1}, x_{2}\right) \rightarrow\left(u_{12}, v=x_{1}\right) \Rightarrow x_{2}=\frac{u_{12}}{v}$ gives $\mathrm{d} x_{1} \wedge \mathrm{d} x_{2}=\frac{1}{v} \mathrm{~d} u_{12} \wedge \mathrm{d} v$. Let $x_{1}>0, x_{2}>0$ be real scalar random variables, independently distributed with density functions $f_{j}\left(x_{j}\right), j=1,2$ respectively, then the joint density is $f_{1}\left(x_{1}\right) f_{2}\left(x_{2}\right)$. Let the density of $u_{12}=x_{1} x_{2}$ be denoted by $g_{12}\left(u_{12}\right)$. Then

$$
\begin{aligned}
g_{12}\left(u_{12}\right) & =\int_{v} \frac{1}{v} f_{1}(v) f_{2}\left(\frac{u_{12}}{v}\right) \mathrm{d} v \\
& =\int_{v} \frac{1}{v} f_{1}\left(\frac{u_{12}}{v}\right) f_{2}(v) \mathrm{d} v .
\end{aligned}
$$

If $f_{1}$ and $f_{2}$ are general functions, may or may not be densities, then (1.3) and (1.4) are called the Mellin convolution of a product. There are two structural integral representations as shown in (1.3) and (1.4). If $f_{1}$ and $f_{2}$ are densities then the Mellin convolution $g_{12}\left(u_{12}\right)$ is also a density, the unique density of the product $x_{1} x_{2}$. If we take the Mellin transform of $g_{12}\left(u_{12}\right)$ or of $g_{21}\left(u_{21}\right)$ we obtain

$$
M_{g_{12}}(s)=M_{f_{1}}(s) M_{f_{2}}(s)=M_{g_{21}}(s)
$$

whenever the Mellin transforms exist. This is the Mellin convolution of a product of two functions property. If

$$
f_{j}\left(x_{j}\right)=c_{j} x_{j}^{\gamma_{j}-1} \mathrm{e}^{-a_{j} x_{j}^{\delta_{j}}}, a_{j}>0, \delta_{j}>0, \gamma_{j}>0, j=1,2,
$$

where $c_{j}$ is the normalizing constant if $f_{j}$ is treated as a density, then, for example, (1.3) is the following:

$$
g_{12}\left(u_{12}\right)=c_{1} c_{2} u_{12}^{\gamma_{2}-1} \int_{v>0} v^{\gamma_{1}-\gamma_{2}-1} \mathrm{e}^{-a v^{\delta_{1}}-a_{2} \frac{u_{12}^{\delta_{2}}}{v^{\delta_{2}}}} \mathrm{~d} v .
$$

The integral in (1.7) is a Mellin convolution of a product, it is the density of a product when $f_{j}, j=1,2$ are densities, and it is connected to many different problems in different areas. For $\delta_{1}=\delta_{2}=1$, it is the basic Krätzel integral, see [2]. For $\delta_{1}=1, \delta_{2}=\frac{1}{2}$ it is the basic reaction-rate probability integral in nuclear reaction rate theory, see [9]. For $\delta_{1}=1, \delta_{2}=1$ the integrand there is the basic inverse Gaussian density. It is a Bessel integral and some people call it a generalized gamma integral. It is the unconditional density in a Bayesian structure where both the conditional and marginal densities belong to exponential densities. For general $\delta_{1}, \delta_{2}$ it is a generalized form of all the above problems in different areas. When $f_{1}\left(x_{1}\right)$ is a real scalar type- 1 beta density with the parameters $(\gamma+1, \alpha)$ or with the density, 


$$
f_{1}\left(x_{1}\right)=\frac{\Gamma(\gamma+1+\alpha)}{\Gamma(\gamma+1) \Gamma(\alpha)} x_{1}^{\gamma}\left(1-x_{1}\right)^{\alpha-1}, 0<x_{1}<1, \gamma>-1, \alpha>0
$$

then (1.4) has the following form:

$$
\begin{aligned}
g_{12}\left(u_{12}\right) & =\frac{\Gamma(\gamma+1+\alpha)}{\Gamma(\gamma+1)} \frac{u_{12}^{\gamma}}{\Gamma(\alpha)} \int_{v>u_{12}} v^{-\gamma-\alpha}\left(v-u_{12}\right)^{\alpha-1} f(v) \mathrm{d} v \\
& =\frac{\Gamma(\gamma+1+\alpha)}{\Gamma(\gamma+1)} K_{2, \gamma}^{-\alpha} f
\end{aligned}
$$

where $f_{2}=f$ is taken as an arbitrary density, and $K_{2, \gamma}^{-\alpha} f$ is the Erdélyi-Kober fractional integral of the second kind of order $\alpha$ and parameter $\gamma$, see [7]. Hence $K_{2, \gamma}^{-\alpha} f$ is a constant multiple of a statistical density $g_{12}$ when $f_{1}$ and $f_{2}=f$ are densities, otherwise the Mellin convolution of a product, $g_{12}$, is connected to Erdélyi-Kober fractional integral of the second kind of order $\alpha$ and parameter $\gamma$. Mellin convolutions of products and ratios of scalar variables are discussed in $[8]$.

In the light of the interesting applications of Mellin convolution of a product, as seen above, we will consider M-convolutions of products and ratios involving three matrices or three functions.

\section{M-convolutions involving three functions}

It is easier to discuss and interpret the problems and results in terms of matrixvariate random variables. Let $X_{j}>O, j=1,2,3$, be $p \times p$ real positive definite matrix random variables, independently distributed with densities $f_{j}\left(X_{j}\right), j=$ $1,2,3$, respectively. Then their joint density is $f_{1}\left(X_{1}\right) f_{2}\left(X_{2}\right) f_{3}\left(X_{3}\right)$. Let $U_{123}=$ $X_{1}^{\frac{1}{2}} X_{2}^{\frac{1}{2}} X_{3} X_{2}^{\frac{1}{2}} X_{1}^{\frac{1}{2}}$ be the symmetric product of the three matrices where $X_{j}^{\frac{1}{2}}$ means the positive definite square root of the real positive definite matrix $X_{j}>O$. When $f_{j}\left(X_{j}\right), j=1,2,3$ are assumed to be symmetric functions, all symmetric forms of the product of $X_{1}, X_{2}, X_{3}$ will have the same density. Hence all symmetric forms of the product of $X_{1}, X_{2}, X_{3}$ will be denoted by $U_{123}, U_{132}, U_{231}$ etc. and their densities denoted by $g_{123}\left(U_{132}\right)$ etc. For fixed $X_{1}$ and $X_{2}$ we have $\mathrm{d} U_{123}=\left|X_{1}\right|^{\frac{p+1}{2}}\left|X_{2}\right|^{\frac{p+1}{2}} \mathrm{~d} X_{3}$ by using a result in [5] on linear transformations. The result is stated here as a lemma, equation (2.2).

Lemma 2.1. Let $A$ be $m \times m$ and $B$ be $n \times n$ nonsingular constant matrices. Let $X=\left(x_{i j}\right)$ and $Y=\left(y_{i j}\right)$ be $m \times n$ real matrices of distinct real elements $x_{i j}$ 's and $y_{i j}$ 's. Then, 


$$
Y=A X B \Rightarrow \mathrm{d} Y=|A|^{n}|B|^{m} \mathrm{~d} X .
$$

When $X=X^{\prime}$ is $p \times p$ real symmetric and if $A$ is a $p \times p$ nonsingular constant matrix, then

$$
Y=A X A^{\prime} \Rightarrow \mathrm{d} Y=|A|^{p+1} \mathrm{~d} X
$$

Let $X_{1}=V_{1}, X_{2}=V_{2}$. Consider the transformation $\left(X_{1}, X_{2}, X_{3}\right) \rightarrow$ $\left(V_{1}, V_{2}, U_{123}\right)$. Then by the repeated application of $(2.2)$ we have

$$
\mathrm{d} X_{1} \wedge \mathrm{d} X_{2} \wedge \mathrm{d} X_{3}=\left|V_{1}\right|^{-\frac{p+1}{2}}\left|V_{2}\right|^{-\frac{p+1}{2}} \mathrm{~d} V_{1} \wedge \mathrm{d} V_{2} \wedge \mathrm{d} U_{123}
$$

Then the density of $U_{123}$, denoted by $g_{123}\left(U_{123}\right)$, is the following:

$$
\begin{aligned}
g_{123}\left(U_{123}\right) & =\int_{V_{1}} \int_{V_{2}}\left|V_{1}\right|^{-\frac{p+1}{2}}\left|V_{2}\right|^{-\frac{p+1}{2}} f_{1}\left(V_{1}\right) f_{2}\left(V_{2}\right) \\
& \times f_{3}\left(V_{2}^{-\frac{1}{2}} V_{1}^{-\frac{1}{2}} U_{123} V_{1}^{-\frac{1}{2}} V_{2}^{-\frac{1}{2}}\right) \mathrm{d} V_{1} \wedge \mathrm{d} V_{2} .
\end{aligned}
$$

Then $g_{123}$ will be called M-convolution of a product of three functions $f_{1}, f_{2}, f_{3}$ when $f_{1}, f_{2}, f_{3}$ are general functions including densities, and when $f_{j}, j=1,2,3$ are densities then this M-convolution of a product is a density of the symmetric product $U_{123}$ also. M-convolution, analogous to the Mellin convolution property for real scalar variables, is that if we take the M-transform or matrix transform of $g_{123}$ then we obtain the product of the M-transforms of $f_{1}, f_{2}, f_{3}$. The Mtransform of a real-valued scalar function $f(X)$ of a $p \times p$ real positive definite matrix $X>O$, with parameter $\rho$, is defined and denoted as

$$
M_{f}(\rho)=\int_{X>O}|X|^{\rho-\frac{p+1}{2}} f(X) \mathrm{d} X, \Re(\rho)>\frac{p-1}{2},
$$

whenever the integral is convergent. A class of functions is defined by (2.5) for a given scalar parameter $\rho$. Let us take the M-transform of $g_{123}$ of (2.4). That is,

$$
M_{g_{123}}(\rho)=\int_{U_{123}>O}\left|U_{123}\right|^{\rho-\frac{p+1}{2}} g_{123}\left(U_{123}\right) \mathrm{d} U_{123} .
$$

Take the integral over $U_{123}$ in $f_{3}$ first, under the condition of interchange of integrals. That is,

$$
\begin{aligned}
& \int_{U_{123}>O}\left|U_{123}\right|^{\rho-\frac{p+1}{2}} f_{3}\left(V_{2}^{-\frac{1}{2}} V_{1}^{-\frac{1}{2}} U_{123} V_{1}^{-\frac{1}{2}} V_{2}^{-\frac{1}{2}}\right) \mathrm{d} U_{123} \\
& =\left|V_{1}\right|^{\rho}\left|V_{2}\right|^{\rho} \int_{T>O}|T|^{\rho-\frac{p+1}{2}} f_{3}(T) \mathrm{d} T, T=V_{2}^{-\frac{1}{2}} V_{1}^{-\frac{1}{2}} U_{123} V_{1}^{-\frac{1}{2}} V_{2}^{-\frac{1}{2}}
\end{aligned}
$$




$$
=\left|V_{1}\right|^{\rho}\left|V_{2}\right|^{\rho} M_{f_{3}}(\rho)
$$

whenever the M-transform exists. The integrals over $V_{1}$ and $V_{2}$ give $M_{f_{1}}(\rho) M_{f_{2}}(\rho)$ whenever the M-transforms exist. This is the M-convolution of the product property. When M-transform and M-convolution were defined in [4], no physical interpretations could be given. Now, M-convolution of a product in (2.4) is given an interpretation as a statistical density of a symmetric product of matrix random variables. More interpretations will be given later. Consider the determinant of $U_{123}$ and take the $\left(\rho-\frac{p+1}{2}\right)$ th moment of the determinant of $U_{123}$. That is, denoting expected value of $(\cdot)$ by $E(\cdot)$, we have the following:

$$
\begin{aligned}
& E\left[\left|U_{123}\right|^{\rho-\frac{p+1}{2}}\right]=E\left[\left|X_{1}^{\frac{1}{2}} X_{2}^{\frac{1}{2}} X_{3} X_{2}^{\frac{1}{2}} X_{1}^{\frac{1}{2}}\right|^{\rho-\frac{p+1}{2}}\right]=E\left[\left|X_{1}\right|\left|X_{2}\right|\left|X_{3}\right|\right]^{\rho-\frac{p+1}{2}} \\
& =E\left[\left|X_{1}\right|^{\rho-\frac{p+1}{2}}\right] E\left[\left|X_{2}\right|^{\rho-\frac{p+1}{2}}\right] E\left[\left|X_{3}\right|^{\rho-\frac{p+1}{2}}\right] \text { due to independence } \\
& =M_{f_{1}}(\rho) M_{f_{2}}(\rho) M_{f_{3}}(\rho),
\end{aligned}
$$

whenever the moments exist, where for example, the $\left(\rho-\frac{p+1}{2}\right)$ th moment of $\left|X_{j}\right|$ is the following:

$$
\int_{X_{j}>O}\left|X_{j}\right|^{\rho-\frac{p+1}{2}} f_{j}\left(X_{j}\right) \mathrm{d} X_{j}=M_{f_{j}}(\rho),
$$

whenever the integral is convergent. Thus, the M-convolution of a product property is also given a physical interpretation in terms of moments of determinants as in (2.7).

We can also establish a connection to fractional integrals. Let $f_{3}$ be a real matrix-variate type- 1 beta density with the parameters $\left(\gamma+\frac{p+1}{2}, \alpha\right)$ or with the density

$$
f_{3}\left(X_{3}\right)=\frac{\Gamma_{p}\left(\gamma+\frac{p+1}{2}+\alpha\right)}{\Gamma_{p}\left(\gamma+\frac{p+1}{2}\right) \Gamma_{p}(\alpha)}\left|X_{3}\right|^{\gamma}\left|I-X_{3}\right|^{\alpha-\frac{p+1}{2}}, 0<X_{3}<I,
$$

for $\gamma>-1, \alpha>\frac{p-1}{2}$. Usually the parameters in a statistical density are real but the gammas in (2.8) also hold for $\Re(\gamma)>-1, \Re(\alpha)>\frac{p-1}{2}$, where, for example, $\Gamma_{p}(\alpha)$ is the real matrix-variate gamma function given by

$$
\Gamma_{p}(\alpha)=\pi^{\frac{p(p-1)}{4}} \Gamma(\alpha) \Gamma\left(\alpha-\frac{1}{2}\right) \ldots \Gamma\left(\alpha-\frac{p-1}{2}\right), \Re(\alpha)>\frac{p-1}{2},
$$

see [5] for its derivation. Consider

$$
\left|V_{1}\right|^{-\frac{p+1}{2}}\left|V_{2}\right|^{-\frac{p+1}{2}} f_{3}\left(V_{2}^{-\frac{1}{2}} V_{1}^{-\frac{1}{2}} U_{123} V_{1}^{-\frac{1}{2}} V_{2}^{-\frac{1}{2}}\right)
$$




$$
\begin{aligned}
& =\frac{\Gamma_{p}\left(\gamma+\frac{p+1}{2}+\alpha\right)}{\Gamma_{p}\left(\gamma+\frac{p+1}{2}\right) \Gamma_{p}(\alpha)}\left|V_{1}\right|^{-\frac{p+1}{2}}\left|V_{2}\right|^{-\frac{p+1}{2}}\left|V_{2}^{-\frac{1}{2}} V_{1}^{-\frac{1}{2}} U_{123} V_{1}^{-\frac{1}{2}} V_{2}^{-\frac{1}{2}}\right|^{\gamma} \\
& \times\left|I-V_{2}^{-\frac{1}{2}} V_{1}^{-\frac{1}{2}} U_{123} V_{1}^{-\frac{1}{2}} V_{2}^{-\frac{1}{2}}\right|^{\alpha-\frac{p+1}{2}} \\
& =\frac{\Gamma_{p}\left(\gamma+\frac{p+1}{2}+\alpha\right)}{\Gamma_{p}\left(\gamma+\frac{p+1}{2}\right)} \frac{\left|U_{123}\right|^{\gamma}}{\Gamma_{p}(\alpha)}\left|V_{1}\right|^{-\alpha-\gamma}\left|V_{2}\right|^{-\alpha-\gamma} \\
& \times\left|V_{1}^{\frac{1}{2}} V_{2} V_{1}^{\frac{1}{2}}-U_{123}\right|^{\alpha-\frac{p+1}{2}}, \quad \Re(\alpha)>\frac{p-1}{2}, \Re(\gamma)>-1 .
\end{aligned}
$$

Then the density in (2.4) reduces to the following:

$$
\begin{aligned}
& g_{123}\left(U_{123}\right)=\frac{\Gamma_{p}\left(\gamma+\frac{p+1}{2}+\alpha\right)}{\Gamma_{p}\left(\gamma+\frac{p+1}{2}\right)} \frac{\left|U_{123}\right|^{\gamma}}{\Gamma_{p}(\alpha)} \iint_{V_{1}^{\frac{1}{2}} V_{2} V_{1}^{\frac{1}{2}}>U_{123}}\left|V_{1}\right|^{-\gamma-\alpha}\left|V_{2}\right|^{-\gamma-\alpha} \\
& \times\left|V_{1}^{\frac{1}{2}} V_{2} V_{1}^{\frac{1}{2}}-U_{123}\right|^{\alpha-\frac{p+1}{2}} f_{1}\left(V_{1}\right) f_{2}\left(V_{2}\right) \mathrm{d} V_{1} \wedge \mathrm{d} V_{2} \\
& =\frac{\Gamma_{p}\left(\gamma+\frac{p+1}{2}+\alpha\right)}{\Gamma_{p}\left(\gamma+\frac{p+1}{2}\right)} K_{2, \gamma}^{-\alpha}\left(f_{1}, f_{2}\right), \quad R e(\gamma)>-1, \Re(\alpha)>\frac{p-1}{2}
\end{aligned}
$$

where (2.10), if $f_{1}$ or $f_{2}$ is absent, and if the variables are real scalar then we have Erdélyi-Kober fractional integral of the second kind of order $\alpha$ and parameter $\gamma$. Then in (2.11), if $f_{1}$ and $V_{1}$ or $f_{2}$ and $V_{2}$ are absent then $K_{2, \gamma}^{-\alpha} f_{1}$ is a ErdélyiKober fractional integral of the second kind of order $\alpha$ and parameter $\gamma$ in the real matrix-variate case as defined in [7]. Hence (2.11) can be considered as an extension of Erdélyi-Kober fractional integral of the second kind of order $\alpha$ and parameter $\gamma$ for two arbitrary functions $f_{1}$ and $f_{2}$ when $f_{1}, f_{2}, f_{3}$ are general functions and it is a constant multiple of a statistical density when $f_{j}, j=1,2,3$ are densities where $f_{1}$ and $f_{2}$ are arbitrary densities and $f_{3}$ is a type-1 beta density as given in (2.8). The form in (2.11) is different from the multiindex fractional integral defined in [1]. For a discussion of fractional integrals in the real scalar case, see for example [3]. But (2.11) is different and two arbitrary functions are involved. If we extend (2.11) to $k$ functions $f_{1}, \ldots, f_{k}$ then the corresponding M-convolution $g_{1 \ldots k}$ of a symmetric product of $X_{1}, \ldots, X_{k}$, the symmetric product can be taken in a large number of ways, will lead to an extension of (2.11) in terms of $k-1$ arbitrary functions and having the structure of Erdélyi-Kober fractional integral of the second kind. The form in (2.11) is also different from the multivariate fractional integrals described in [7]. Thus, (2.11) is another physical interpretation of a M-convolution of a product as a matrix-variate fractional integral.

By taking different symmetric forms of the product of $X_{1}, X_{2}, X_{3}$ we can obtain other structural representations. 


\subsection{Other structural representation of the densities of symmetric products}

We can obtain different integral representations for the symmetric product of $X_{j}, j=1,2,3$ by considering the following:

$$
X_{1}^{\frac{1}{2}} X_{3}^{\frac{1}{2}} X_{2} X_{3}^{\frac{1}{2}} X_{1}^{\frac{1}{2}}, X_{2}^{\frac{1}{2}} X_{1}^{\frac{1}{2}} X_{3} X_{1}^{\frac{1}{2}} X_{2}^{\frac{1}{2}}, X_{2}^{\frac{1}{3}} X_{3}^{\frac{1}{2}} X_{1} X_{3}^{\frac{1}{2}} X_{1}^{\frac{1}{2}} \text {, etc. }
$$

We can also obtain other integral representations by using symmetric product of two matrices two times. For example, take $U_{123}=X_{1}^{\frac{1}{2}}\left(X_{2}^{\frac{1}{2}} X_{3} X_{2}^{\frac{1}{2}}\right) X_{1}^{\frac{1}{2}}=$ $X_{1}^{\frac{1}{2}} U_{23} X_{1}^{\frac{1}{2}}, U_{23}=X_{2}^{\frac{1}{2}} X_{3} X_{2}^{\frac{1}{2}}$. Compute the density of $U_{23}$, namely, $g_{23}\left(U_{23}\right)$ first. There are two structural representations here for $g_{23}$. There are two structural representations for $U_{123}=X_{1}^{\frac{1}{2}} U_{23} X_{1}^{\frac{1}{2}}$. Thus, four different integral representations are available for each of the symmetric product above, some of the integral representations may coincide. All these M-convolutions will give the same M-transform as $M_{f_{1}}(\rho) M_{f_{2}}(\rho) M_{f_{3}}(\rho)$ whenever the M-transforms exist.

\section{Structural representations of symmetric ratio}

Consider three $p \times p$ real positive definite matrix random variables $X_{j}, j=1,2,3$, independently distributed with densities $f_{j}\left(X_{j}\right), j=1,2,3$, respectively. Let us consider a structure where $X_{2}$ and $X_{3}$ are in the numerator and $X_{1}$ is in the denominator, denoted by $U_{23 / 1}$. Let $U_{23 / 1}=X_{1}^{-\frac{1}{2}} X_{2}^{\frac{1}{2}} X_{3} X_{2}^{\frac{1}{2}} X_{1}^{-\frac{1}{2}}$. Since inverses are involved, in order to compute the Jacobian we will need one more result from [5] which will be presented here as a lemma.

Lemma 3.1. Let $X=\left(x_{i j}\right)$ be a $p \times p$ real nonsingular matrix and let $Y=X^{-1}$. Then if the $x_{i j}$ 's are all distinct or distinct real scalar variables, then

$$
Y=X^{-1} \Rightarrow \mathrm{d} Y=|X|^{-2 p} \mathrm{~d} X
$$

and if $X=X^{\prime}$ (symmetric) and of distinct $p(p+1) / 2$ scalar elements, then

$$
Y=X^{-1} \Rightarrow \mathrm{d} Y=|X|^{-(p+1)} \mathrm{d} X
$$

In $U_{23 / 1}$ let $V_{1}=X_{1}, V_{2}=X_{2}$ that is, $X_{3}=V_{2}^{-\frac{1}{2}} V_{1}^{\frac{1}{2}} U_{23 / 1} V_{1}^{\frac{1}{2}} V_{2}^{-\frac{1}{2}}$ and from Lemma 2.1,

$$
\mathrm{d} X_{1} \wedge \mathrm{d} X_{2} \wedge \mathrm{d} X_{3}=\left|V_{1}\right|^{\frac{p+1}{2}}\left|V_{2}\right|^{-\frac{p+1}{2}} \mathrm{~d} V_{1} \wedge \mathrm{d} V_{2} \wedge \mathrm{d} U_{23 / 1}
$$

If the density of $U_{23 / 1}$ is denoted as $g_{23 / 1}\left(U_{23 / 1}\right)$, then 


$$
\begin{aligned}
g_{23 / 1}\left(U_{23 / 1}\right) & =\int_{V_{1}} \int_{V_{2}}\left|V_{1}\right|^{\frac{p+1}{2}}\left|V_{2}\right|^{-\frac{p+1}{2}} f_{1}\left(V_{1}\right) f_{2}\left(V_{2}\right) \\
& \times f_{3}\left(V_{2}^{-\frac{1}{2}} V_{1}^{\frac{1}{2}} U_{23 / 1} V_{1}^{\frac{1}{2}} V_{2}^{-\frac{1}{2}}\right) \mathrm{d} V_{1} \wedge \mathrm{d} V_{2} .
\end{aligned}
$$

The integral representation in (3.3) is called a M-convolution of a ratio involving three functions when $f_{1}, f_{2}, f_{3}$ are general real-valued scalar functions and the M-convolution is the unique density when $f_{j} \cdot j=1,2,3$ are densities. Let us take the M-transform of $g_{23 / 1}$, with parameter $\rho$,

$$
M_{g_{23 / 1}}(\rho)=\int_{U_{23 / 1}}\left|U_{23 / 1}\right|^{\rho-\frac{p+1}{2}} g_{23 / 1}\left(U_{23 / 1}\right) \mathrm{d} U_{23 / 1}, \Re(\rho)>\frac{p-1}{2} .
$$

Take the M-transform over $U_{23 / 1}$ in $f_{3}$ first under the condition of interchange of integrals. Then

$$
\begin{aligned}
& \int_{U_{23 / 1}>O}\left|U_{23 / 1}\right|^{\rho-\frac{p+1}{2}} f_{3}\left(V_{2}^{-\frac{1}{2}} V_{1}^{\frac{1}{2}} U_{23 / 1} V_{1}^{\frac{1}{2}} V_{2}^{-\frac{1}{2}}\right) \mathrm{d} U_{23 / 1} \\
& =\left|V_{1}\right|^{-\rho}\left|V_{2}\right|^{\rho} \int_{T>O}|T|^{\rho-\frac{p+1}{2}} f_{3}(T) \mathrm{d} T, T=V_{2}^{-\frac{1}{2}} V_{1}^{-\frac{1}{2}} U_{23 / 1} V_{1}^{\frac{1}{2}} V_{2}^{-\frac{1}{2}} \\
& =\left|V_{1}\right|^{-\rho}\left|V_{2}\right|^{\rho} M_{f_{3}}(\rho),
\end{aligned}
$$

whenever the integral is convergent.

Then the integral over $V_{2}$ in $f_{2}$ gives $M_{f_{2}}(\rho)$ whenever the M-transform exists. The integral over $V_{1}$ in $f_{1}$ gives

$$
\begin{aligned}
& \int_{V_{1}>O}\left|V_{1}\right|^{-\rho+\frac{p+1}{2}} f_{2}\left(V_{2}\right) \mathrm{d} V_{1} \\
= & \int_{V_{1}>O}\left|V_{1}\right|^{(p+1-\rho)-\frac{p+1}{2}} f_{2}\left(V_{1}\right) \mathrm{d} V_{1}=M_{f_{1}}(p+1-\rho),
\end{aligned}
$$

whenever the integral is convergent. That is,

$$
M_{U_{23 / 1}}(\rho)=M_{f_{1}}(p+1-\rho) M_{f_{2}}(\rho) M_{f_{3}}(\rho)
$$

whenever the M-transforms exist. When $f_{j}, j=1,2,3$ are densities we can also see the result in (3.4) by using the expected values of the determinant of $U_{23 / 1}$,

$$
\begin{aligned}
& E\left[\left|U_{23 / 1}\right|^{\rho-\frac{p+1}{2}}\right]=E\left[\left|X_{1}\right|^{-\frac{1}{2}}\left|X_{2}\right|^{\frac{1}{2}}\left|X_{3}\right|\left|X_{2}\right|^{\frac{1}{2}}\left|X_{1}\right|^{-\frac{1}{2}}\right]^{\rho-\frac{p+1}{2}} \\
& =E\left[\left|X_{1}\right|^{-\rho+\frac{p+1}{2}}\right] E\left[\left|X_{2}\right|^{\rho-\frac{p+1}{2}}\right] E\left[\left|X_{3}\right|^{\rho-\frac{p+1}{2}}\right] \text { due to independence }
\end{aligned}
$$




$$
=M_{f_{1}}(p+1-\rho) M_{f_{2}}(\rho) M_{f_{3}}(\rho),
$$

whenever the M-transforms exist.

From this it is clear that all symmetric ratios with $X_{2}, X_{3}$ in the numerator and $X_{1}$ in the denominator will give rise to the same M-transform in (3.4). If $X_{2}$ and $X_{3}$ are interchanged in $U_{23 / 1}$ or if we consider $U_{32 / 1}$ then the roles of $f_{2}$ and $f_{3}$ will interchange and there will not be any structural change in (3.3). But note that

$$
U_{23 / 1}=X_{1}^{-\frac{1}{2}}\left(X_{2}^{\frac{1}{2}} X_{3} X_{2}^{\frac{1}{2}}\right) X_{1}^{-\frac{1}{2}}=X_{1}^{-\frac{1}{2}} U_{23} X_{1}^{-\frac{1}{2}}, U_{23}=X_{2}^{\frac{1}{2}} X_{3} X_{2}^{\frac{1}{2}} .
$$

Then the density of $U_{23}$, denoted by $g_{23}$ is available from the M-convolution of a product of two functions $f_{2}$ and $f_{3}$. Now, looking at $U_{23 / 1}$ as a symmetric product let $V_{1}=X_{1}$. Then $U_{23}=V_{1}^{\frac{1}{2}} U_{23 / 1} V_{1}^{\frac{1}{2}}$ and $\mathrm{d} X_{1} \wedge \mathrm{d} U_{23}=\left|V_{1}\right|^{\frac{p+1}{2}} \mathrm{~d} V_{1} \wedge$ $\mathrm{d} U_{23 / 1}$. Then

$$
\begin{aligned}
& g_{23 / 1}\left(U_{23 / 1}\right)=\int_{V_{1}}\left|V_{1}\right|^{\frac{p+1}{2}} f_{1}\left(V_{1}\right) g_{23}\left(V_{1}^{\frac{1}{2}} U_{23 / 1} V_{1}^{\frac{1}{2}}\right) \mathrm{d} V_{1} \\
& =\int_{V_{1}}\left|V_{1}\right|^{\frac{p+1}{2}} f_{1}\left(V_{1}\right)\left\{\int_{V_{2}}\left|V_{2}\right|^{-\frac{p+1}{2}} f_{2}\left(V_{2}\right) f_{3}\left(V_{2}^{-\frac{1}{2}} V_{1}^{\frac{1}{2}} U_{23 / 1} V_{1}^{\frac{1}{2}} V_{2}^{-\frac{1}{2}}\right) \mathrm{d} V_{2}\right\} \mathrm{d} V_{1} \\
& =\int_{V_{1}} \int_{V_{1}}\left|V_{1}\right|^{\frac{p+1}{2}}\left|V_{2}\right|^{-\frac{p+1}{2}} f_{1}\left(V_{1}\right) f_{2}\left(V_{2}\right) \\
& \times f_{3}\left(V_{2}^{-\frac{1}{2}} V_{1}^{\frac{1}{2}} U_{23 / 1} V_{1}^{\frac{1}{2}} V_{2}^{-\frac{1}{2}}\right) \mathrm{d} V_{1} \wedge \mathrm{d} V_{2}
\end{aligned}
$$

which is of the structure in (3.3). Hence, no new structure is involved. If $U_{23 / 1}$ is taken as $U_{23 / 1}=U_{23}^{\frac{1}{2}} X_{1}^{-1} U_{23}^{\frac{1}{2}}$ observing that $f_{j}, j=1,2,3$ are assumed to be symmetric functions and hence the values of the functions remain the same if the form $X_{1}^{-\frac{1}{2}} U_{23} X_{1}^{-\frac{1}{2}}$ or the form $U_{23}^{\frac{1}{2}} X_{1}^{-1} U_{23}^{\frac{1}{2}}$ is taken. Let $U_{23}=V_{2}$, then $X_{1}=V_{2}^{\frac{1}{2}} U_{23 / 1}^{-1} V_{2}^{\frac{1}{2}}$ and

$$
\mathrm{d} X_{1} \wedge \mathrm{d} U_{23}=\left|V_{2}\right|^{\frac{p+1}{2}}\left|U_{23 / 1}\right|^{-(p+1)} \mathrm{d} V_{2} \wedge \mathrm{d} U_{23 / 1}
$$

from Lemmas 2.1 and 3.1. Then the density $g_{23 / 1}\left(U_{23 / 1}\right)$ is the following:

$$
\begin{aligned}
g_{23 / 1}\left(U_{23 / 1}\right) & =\int_{V_{2}}\left|V_{2}\right|^{\frac{p+1}{2}}\left|U_{23 / 1}\right|^{-(p+1)} g_{23}\left(V_{2}\right) f_{1}\left(V_{2}^{\frac{1}{2}} U_{23 / 1}^{-1} V_{2}^{\frac{1}{2}}\right) \mathrm{d} V_{2} \\
& =\int_{V_{2}}\left|V_{2}\right|^{\frac{p+1}{2}}\left|U_{23 / 1}\right|^{-(p+1)} f_{1}\left(V_{2}^{\frac{1}{2}} U_{23 / 1}^{-1} V_{2}^{\frac{1}{2}}\right) \\
& \times\left\{\int_{V_{1}}\left|V_{1}\right|^{-\frac{p+1}{2}} f_{2}\left(V_{1}\right) f_{3}\left(V_{1}^{-\frac{1}{2}} V_{2} V_{1}^{-\frac{1}{2}}\right) \mathrm{d} V_{1}\right\} \mathrm{d} V_{2}
\end{aligned}
$$




$$
\begin{aligned}
& =\int_{V_{1}} \int_{V_{2}}\left|V_{1}\right|^{-\frac{p+1}{2}}\left|V_{2}\right|^{\frac{p+1}{2}}\left|U_{23 / 1}\right|^{-(p+1)} f_{1}\left(V_{2}^{\frac{1}{2}} U_{23 / 1}^{-1} V_{2}^{\frac{1}{2}}\right) \\
& \times f_{2}\left(V_{1}\right) f_{3}\left(V_{1}^{-\frac{1}{2}} V_{2} V_{1}^{-\frac{1}{2}}\right) \mathrm{d} V_{1} \wedge \mathrm{d} V_{2} .
\end{aligned}
$$

Observe that (3.5) is a new structure compared to (3.3). This (3.5) is a Mconvolution of a ratio involving three functions. It is easy to see that the M-transform in (3.5) gives rise to (3.4). To this end, take the M-transform with respect to $U_{23 / 1}$ in $f_{1}$ first. Then integrate over $V_{2}$ and then over $V_{1}$ to see the result. Let

$$
U_{23 / 1}=X_{2}^{\frac{1}{2}} X_{3}^{\frac{1}{2}} X_{1}^{-1} X_{3}^{\frac{1}{2}} X_{2}^{\frac{1}{2}} \Rightarrow X_{1}=X_{3}^{\frac{1}{2}} X_{2}^{\frac{1}{2}} U_{23 / 1}^{-1} X_{2}^{\frac{1}{2}} X_{3}^{\frac{1}{2}}
$$

Let $V_{1}=X_{2}, V_{2}=X_{3}$. Then

$$
\mathrm{d} X_{1} \wedge \mathrm{d} X_{2} \wedge \mathrm{d} X_{3}=\left|V_{1}\right|^{\frac{p+1}{2}}\left|V_{2}\right|^{\frac{p+1}{2}}\left|U_{23 / 1}\right|^{-(p+1)} \mathrm{d} V_{1} \wedge \mathrm{d} V_{2} \wedge \mathrm{d} U_{23 / 1},
$$

from Lemmas 2.1 and 3.1. Then the density $g_{23 / 1}\left(U_{23 / 1}\right)$ is given by the following:

$$
\begin{aligned}
g_{23 / 1}\left(U_{23 / 1}\right) & =\int_{V_{1}} \int_{V_{2}}\left|V_{1}\right|^{\frac{p+1}{2}}\left|V_{2}\right|^{\frac{p+1}{2}}\left|U_{23 / 1}\right|^{-(p+1)} f_{1}\left(V_{2}^{\frac{1}{2}} V_{1}^{\frac{1}{2}} U_{23 / 1}^{-1} V_{1}^{\frac{1}{2}} V_{2}^{\frac{1}{2}}\right) \\
& \times f_{2}\left(V_{1}\right) f_{3}\left(V_{2}\right) \mathrm{d} V_{1} \wedge \mathrm{d} V_{2} .
\end{aligned}
$$

Note that (3.6) is different from (3.3) and (3.5) and hence it is a new representation for the $\mathrm{M}$-convolution of a ratio of three functions $f_{j}, j=1,2,3$. If we interchange $V_{1}$ and $V_{2}$ then the roles of $f_{2}$ and $f_{3}$ change but no new structure is obtained. Again, we can see that the M-transform of in (3.6) also gives (3.4). To this end, take the M-transform of $U_{23 / 1}$ in $f_{1}$ first to obtain $\left|V_{1}\right|^{\rho-(p+1)}\left|V_{2}\right|^{\rho-(p+1)} M_{f_{1}}(p+1-\rho)$ when this M-transform exists. Now, integrate over $V_{1}$ and $V_{2}$ to see the result.

\subsection{Some practical situations}

Let $f_{j}, j=1,2,3$ be real matrix-variate gamma densities

$$
f_{j}\left(X_{j}\right)=C_{j}\left|X_{j}\right|^{\gamma_{j}-\frac{p+1}{2}} \mathrm{e}^{-a_{j} \operatorname{tr}\left(X_{j}\right)}, X_{j}>0, a_{j}>0, \gamma_{j}>\frac{p-1}{2},
$$

$j=1,2,3$, where $C_{j}$ is the normalizing constant. Then if we consider $(3.3)$ structure then in the exponent we have $V_{2}$ with positive and negative exponents and $V_{1}$ with positive exponent. This is a matrix-variate extension of Krätzel integral, unconditional density in Bayesian structure, inverse Gaussian form etc. In (3.5) structure, $V_{1}$ will have both negative and positive xponents and $V_{2}$ 
will have positive exponent. In (3.6) structure, both $V_{1}$ and $V_{2}$ will have only positive exponents. This form can be integrated out with respect to $V_{1}$ and $V_{2}$, giving rise to an extended real matrix-variate type- 2 beta form.

Let $X_{1}$ have a real matrix-variate type-1 beta density with the parameters $(\gamma, \alpha)$ or with the density

$$
f_{1}\left(X_{1}\right)=\frac{\Gamma_{p}(\gamma+\alpha)}{\Gamma_{p}(\gamma) \Gamma_{p}(\alpha)}\left|X_{1}\right|^{\gamma-\frac{p+1}{2}}\left|I-X_{1}\right|^{\alpha-\frac{p+1}{2}}
$$

$0<X_{1}<I, \alpha>\frac{p-1}{2}, \gamma>\frac{p-1}{2}$. Then from (3.6)

$$
\begin{aligned}
& \left|V_{1}\right|^{\frac{p+1}{2}}\left|V_{2}\right|^{\frac{p+1}{2}} f_{1}\left(V_{2}^{\frac{1}{2}} V_{1}^{\frac{1}{2}} U_{23 / 1}^{-1} V_{1}^{\frac{1}{2}} V_{2}^{\frac{1}{2}}\right)\left|U_{23 / 1}\right|^{-(p+1)} \\
& =\frac{\Gamma_{p}(\gamma+\alpha)}{\Gamma_{p}(\gamma) \Gamma_{p}(\alpha)}\left|V_{1}\right|^{\gamma}\left|V_{2}\right|^{\gamma}\left|U_{23 / 1}\right|^{-\gamma-\alpha}\left|U_{23 / 1}-V_{1}^{\frac{1}{2}} V_{2} V_{1}^{\frac{1}{2}}\right|^{\alpha-\frac{p+1}{2}} .
\end{aligned}
$$

Then (3.6) has the following form:

$$
\begin{aligned}
g_{23 / 1}\left(U_{23 / 1}\right) & =\frac{\Gamma_{p}(\gamma+\alpha)}{\Gamma_{p}(\gamma)} \frac{\left|U_{23 / 1}\right|^{-\gamma-\alpha}}{\Gamma_{p}(\alpha)} \iint_{V_{1}^{\frac{1}{2}} V_{2} V_{1}^{\frac{1}{2}}<U_{23 / 1}}\left|V_{1}\right|^{\gamma}\left|V_{2}\right|^{\gamma} \\
& \times\left|U_{23 / 1}-V_{1}^{\frac{1}{2}} V_{2} V_{1}^{\frac{1}{2}}\right|^{\alpha-\frac{p+1}{2}} f_{2}\left(V_{1}\right) f_{3}\left(V_{2}\right) \mathrm{d} V_{1} \wedge \mathrm{d} V_{2} \\
& =\frac{\Gamma_{p}(\gamma+\alpha)}{\Gamma_{p}(\gamma)} K_{1, \gamma}^{-\alpha}\left(f_{2}, f_{3}\right)
\end{aligned}
$$

where $K_{1, \gamma}^{-\alpha}\left(f_{1}, f_{2}\right)$ is an extended form of Erdélyi-Kober fractional integral of the first kind of order $\alpha$ and parameter $\gamma$ on two arbitrary functions $f_{2}, f_{3}$, in the real matrix-variate case. If $f_{2}$ and $V_{1}$ or $f_{3}$ and $V_{2}$ are absent then we have $K_{1, \gamma}^{-\alpha} f_{3}$ or $f_{2}$ which is a real matrix-variate Erdélyi-Kober fractional integral of the first kind of order $\alpha$ and parameter $\gamma$, see [7]. Hence (3.8) gives an extension to a two arbitrary function format, which in the real scalar case is different from the multi-index fractional integral of [1]. The following are the integral structures for $g_{23 / 1}$ or when $X_{2}$ and $X_{3}$ are in the numerator and $X_{1}$ is in the denominator,

$$
\begin{gathered}
g_{23 / 1}\left(U_{23 / 1}\right)=\int_{V_{1}} \int_{V_{2}}\left|V_{1}\right|^{\frac{p+1}{2}}\left|V_{2}\right|^{-\frac{p+1}{2}} f_{1}\left(V_{1}\right) f_{2}\left(V_{2}\right) \\
\times f_{3}\left(V_{2}^{-\frac{1}{2}} V_{1}^{\frac{1}{2}} U_{23 / 1} V_{1}^{\frac{1}{2}} V_{2}^{-\frac{1}{2}}\right) \mathrm{d} V_{1} \wedge \mathrm{d} V_{2}, \\
g_{23 / 1}\left(U_{23 / 1}\right)=\int_{V_{1}} \int_{V_{2}}\left|V_{1}\right|^{-\frac{p+1}{2}}\left|V_{2}\right|^{\frac{p+1}{2}}\left|U_{23 / 1}\right|^{-(p+1)} f_{1}\left(V_{2}^{\frac{1}{2}} U_{23 / 1}^{-1} V_{2}^{\frac{1}{2}}\right)
\end{gathered}
$$




$$
\begin{aligned}
& \times f_{2}\left(V_{1}\right) f_{3}\left(V_{1}^{-\frac{1}{2}} V_{2} V_{1}^{-\frac{1}{2}}\right) \mathrm{d} V_{1} \wedge \mathrm{d} V_{2} \\
g_{23 / 1}\left(U_{23 / 1}\right)= & \int_{V_{1}} \int_{V_{2}}\left|V_{1}\right|^{\frac{p+1}{2}}\left|V_{2}\right|^{\frac{p+1}{2}}\left|U_{23 / 1}\right|^{-(p+1)} f_{1}\left(V_{2}^{\frac{1}{2}} V_{1}^{\frac{1}{2}} U_{23 / 1}^{-1} V_{1}^{\frac{1}{2}} V_{2}^{\frac{1}{2}}\right) \\
\times & f_{2}\left(V_{1}\right) f_{3}\left(V_{2}\right) \mathrm{d} V_{1} \wedge \mathrm{d} V_{2} \\
g_{23 / 1}\left(U_{23 / 1}\right) & =\int_{V_{1}} \int_{V_{2}}\left|V_{1}\right|^{-\frac{p+1}{2}}\left|V_{2}\right|^{\frac{p+1}{2}} f_{2}\left(V_{1}^{-\frac{1}{2}} V_{2}^{\frac{1}{2}} U_{23 / 1} V_{2}^{\frac{1}{2}} V_{1}^{-\frac{1}{2}}\right) \\
& \times f_{3}\left(V_{1}\right) f_{2}\left(V_{2}\right) \mathrm{d} V_{1} \wedge \mathrm{d} V_{2} .
\end{aligned}
$$

If we have $X_{1}$ and $X_{2}$ in the numerator and $X_{3}$ in the denominator $U_{12 / 3}$, then the density denoted by $g_{12 / 3}\left(U_{12 / 3}\right)$, has the following forms:

$$
\begin{aligned}
g_{12 / 3}\left(U_{12 / 3}\right)= & \int_{V_{1}} \int_{V_{2}}\left|V_{1}\right|^{-\frac{p+1}{2}}\left|V_{2}\right|^{\frac{p+1}{2}} f_{1}\left(V_{1}\right) f_{3}\left(V_{2}\right) \\
& \times f_{2}\left(V_{1}^{-\frac{1}{2}} V_{2}^{\frac{1}{2}} U_{12 / 3} V_{2}^{\frac{1}{2}} V_{1}^{-\frac{1}{2}}\right) \mathrm{d} V_{1} \wedge \mathrm{d} V_{2} \\
g_{12 / 3}\left(U_{12 / 3}\right)= & \int_{V_{1}} \int_{V_{2}}\left|V_{1}\right|^{-\frac{p+1}{2}}\left|V_{2}\right|^{\frac{p+1}{2}} f_{1}\left(V_{1}^{-\frac{1}{2}} V_{2}^{\frac{1}{2}} U_{12 / 3} V_{2}^{\frac{1}{2}} V_{1}^{-\frac{1}{2}}\right) \\
& \times f_{2}\left(V_{1}\right) f_{3}\left(V_{2}\right) \mathrm{d} V_{1} \wedge \mathrm{d} V_{2}, \\
g_{12 / 3}\left(U_{12 / 3}\right)= & \int_{V_{1}} \int_{V_{2}}\left|V_{1}\right|^{\frac{p+1}{2}}\left|V_{2}\right|^{\frac{p+1}{2}}\left|U_{12 / 3}\right|^{-(p+1)} f_{1}\left(V_{1}\right) f_{2}\left(V_{2}\right) \\
& \times f_{3}\left(V_{2}^{\frac{1}{2}} V_{1}^{\frac{1}{2}} U_{12 / 3}^{-1} V_{1}^{\frac{1}{2}} V_{2}^{\frac{1}{2}}\right) \mathrm{d} V_{1} \wedge \mathrm{d} V_{2}, \\
g_{12 / 3}\left(U_{12 / 3}\right)= & \int_{V_{1}} \int_{V_{2}}\left|V_{1}\right|^{-\frac{p+1}{2}}\left|V_{2}\right|^{\frac{p+1}{2}}\left|U_{12 / 3}\right|^{-(p+1)} f_{3}\left(V_{2}^{\frac{1}{2}} U_{12 / 3}^{-1} V_{2}^{\frac{1}{2}}\right) \\
\times & f_{1}\left(V_{1}\right) f_{2}\left(V_{1}^{-\frac{1}{2}} V_{2} V_{1}^{-\frac{1}{2}}\right) \mathrm{d} V_{1} \wedge \mathrm{d} V_{2} .
\end{aligned}
$$

If we have $X_{1}$ and $X_{3}$ in the numerator and $X_{2}$ in the denominator or $U_{13 / 2}$ with the density $g_{13 / 2}\left(U_{13 / 2}\right)$ then we have the following:

$$
g_{13 / 2}\left(U_{13 / 2}\right)=\int_{V_{1}} \int_{V_{2}}\left|V_{1}\right|^{-\frac{p+1}{2}}\left|V_{2}\right|^{\frac{p+1}{2}} f_{1}\left(V_{1}\right) f_{2}\left(V_{2}\right)
$$




$$
\begin{aligned}
& \times f_{3}\left(V_{1}^{-\frac{1}{2}} V_{2}^{\frac{1}{2}} U_{13 / 2} V_{2}^{\frac{1}{2}} V_{1}^{-\frac{1}{2}}\right) \mathrm{d} V_{1} \wedge \mathrm{d} V_{2}, \\
g_{13 / 2}\left(U_{13 / 2}\right)= & \int_{V_{1}} \int_{V_{2}}\left|V_{1}\right|^{-\frac{p+1}{2}}\left|V_{2}\right|^{\frac{p+1}{2}} f_{1}\left(V_{1}^{-\frac{1}{2}} V_{2}^{\frac{1}{2}} U_{13 / 2} V_{2}^{\frac{1}{2}} V_{1}^{-\frac{1}{2}}\right) \\
\times & f_{3}\left(V_{1}\right) f_{3}\left(V_{2}\right) \mathrm{d} V_{1} \wedge \mathrm{d} V_{2}, \\
g_{13 / 2}\left(U_{13 / 2}\right)= & \int_{V_{1}} \int_{V_{2}}\left|V_{1}\right|^{\frac{p+1}{2}}\left|V_{2}\right|^{\frac{p+1}{2}}\left|U_{13 / 2}\right|^{-(p+1)} f_{1}\left(V_{1}\right) f_{3}\left(V_{2}\right) \\
& \times f_{2}\left(V_{2}^{\frac{1}{2}} V_{1}^{\frac{1}{2}} U_{13 / 2}^{-1} V_{1}^{\frac{1}{2}} V_{2}^{\frac{1}{2}}\right) \mathrm{d} V_{1} \wedge \mathrm{d} V_{2}, \\
g_{13 / 2}\left(U_{13 / 2}\right)= & \int_{V_{1}} \int_{V_{2}}\left|V_{1}\right|^{-\frac{p+1}{2}}\left|V_{2}\right|^{\frac{p+1}{2}}\left|U_{13 / 2}\right|^{-(p+1)} f_{2}\left(V_{2}^{\frac{1}{2}} U_{13 / 2}^{-1} V_{2}^{\frac{1}{2}}\right) \\
\times & f_{1}\left(V_{1}\right) f_{3}\left(V_{1}^{-\frac{1}{2}} V_{2} V_{1}^{-\frac{1}{2}}\right) \mathrm{d} V_{1} \wedge \mathrm{d} V_{2} .
\end{aligned}
$$

In all the integral representation in $g_{12 / 3}$ the M-transform, with parameter $\rho$, is given by

$$
M_{g_{12 / 3}}(\rho)=M_{f_{1}}(\rho) M_{f_{2}}(\rho) M_{f_{3}}(p+1-\rho)
$$

whenever the M-transforms exist. In all the integral representations of $g_{13 / 2}$ the M-transform is given by

$$
M_{g_{13 / 2}}(\rho)=M_{f_{1}}(\rho) M_{f_{2}}(p+1-\rho) M_{f_{3}}(\rho)
$$

whenever the M-transforms exist. In all the integral representations of $g_{23 / 1}$ the M-transform is given by

$$
M_{g_{23 / 1}}(\rho)=M_{f_{1}}(p+1-\rho) M_{f_{2}}(\rho) M_{f_{3}}(\rho)
$$

whenever the M-transforms exist.

\section{Ratios with one variable in the numerator and two variables in the denominator}

We will start with the real scalar variable case. Again, it is easier to interpret in terms of random variables. Let $x_{j}>0, j=1,2,3$, be three real scalar positive random variables, with density functions $f_{j}\left(x_{j}\right), j=1,2,3$, respectively and independently distributed so that the joint density is $f_{1}\left(x_{1}\right) f_{2}\left(x_{2}\right) f_{3}\left(x_{3}\right)$. We will 
consider some ratios involving all the three variables. Let $u_{1 / 23}=\frac{x_{1}}{x_{2} x_{3}}, u_{2 / 13}=$ $\frac{x_{2}}{x_{1} x_{3}}, u_{3 / 12}=\frac{x_{3}}{x_{1} x_{2}}, u_{/ 123}=\frac{1}{x_{1} x_{2} x_{3}}$. In Sections 2 and 3 we have already considered ratios where one variable, scalar or matrix, in the denominator. Note that $u_{/ 123}=\frac{1}{u_{123}}$ and the case $u_{123}$ is already discussed earlier and hence the properties of $u_{/ 123}$ follow from those of $u_{123}$. Since the $(s-1)$ th moments are the Mellin transforms of $f_{j}$ 's with Mellin parameter $s$, we can obtain the Mellin transforms of the densities of all the above variables. Let $g_{1 / 23}$ be the density of $u_{1 / 23}$. Then we have

$$
E\left[u_{1 / 23}\right]^{s-1}=E\left[\frac{x_{1}}{x_{2} x_{3}}\right]^{s-1}=E\left[x_{1}\right]^{s-1} E\left[x_{2}\right]^{-s+1} E\left[x_{3}\right]^{-s+1},
$$

due to independence. Therefore,

$$
M_{g_{1 / 23}}(s)=M_{f_{1}}(s) M_{f_{2}}(2-s) M_{f_{3}}(2-s)
$$

whenever the Mellin transforms exist. Note that (4.1) holds even if $f_{j}$ 's are densities or not and hence $g_{1 / 23}$ is called a Mellin convolution of a ratio involving three functions. In $g_{/ 123}$ we have $M_{g_{/ 123}}(s)=M_{f_{1}}(2-s) M_{f_{2}}(2-s) M_{f_{3}}(2-s)$ whenever the Mellin transforms exist.

Our interest here is to consider the corresponding real matrix-variate cases and their M-convolutions of ratios involving three functions where one matrix is in the numerator and two matrices are in the denominator. Let $X_{j}>O, j=$ $1,2,3$, be $p \times p$ real matrix-variate random variables with densities $f_{j}\left(X_{j}\right), j=$ $1,2,3$ and independently distributed. Consider the symmetric ratio

$$
\begin{gathered}
U_{1 / 23}=X_{2}^{-\frac{1}{2}} X_{3}^{-\frac{1}{2}} X_{1} X_{3}^{-\frac{1}{2}} X_{2}^{-\frac{1}{2}} \\
V_{1}=X_{2}, V_{2}=X_{3} \Rightarrow X_{1}=V_{2}^{\frac{1}{2}} V_{1}^{\frac{1}{2}} U_{1 / 23} V_{1}^{\frac{1}{2}} V_{2}^{\frac{1}{2}} .
\end{gathered}
$$

Then

$$
\mathrm{d} X_{1} \wedge \mathrm{d} X_{2} \wedge \mathrm{d} X_{3}=\left|V_{1}\right|^{\frac{p+1}{2}}\left|V_{2}\right|^{\frac{p+1}{2}} \mathrm{~d} V_{1} \wedge \mathrm{d} V_{2} \wedge \mathrm{d} U_{1 / 23}
$$

from Lemma 2.1. Let the density of $U_{1 / 23}$ be denoted by $g_{1 / 23}\left(U_{1 / 23}\right)$. Then

$$
\begin{aligned}
g_{1 / 23}\left(U_{1 / 23}\right) & =\int_{V_{1}} \int_{V_{2}}\left|V_{1}\right|^{\frac{p+1}{2}}\left|V_{2}\right|^{\frac{p+1}{2}} f_{2}\left(V_{1}\right) f_{3}\left(V_{2}\right) \\
& \times f_{1}\left(V_{2}^{\frac{1}{2}} V_{1}^{\frac{1}{2}} U_{1 / 23} V_{1}^{\frac{1}{2}} V_{2}^{\frac{1}{2}}\right) \mathrm{d} V_{1} \wedge \mathrm{d} V_{2}
\end{aligned}
$$

This (4.2) is the M-convolution of a ratio involving three functions $f_{j}, j=1,2,3$ for general $f_{j}$ 's and it is also a density when $f_{j}, j=1,2,3$ are densities. By using the expected values we have

$$
E\left[\left|U_{1 / 23}\right|\right]^{\rho-\frac{p+1}{2}}=E\left[\mid X_{2}^{-\frac{1}{2}} X_{3}^{-\frac{1}{2}} X_{1} X_{3}^{-\frac{1}{2}} X_{2}^{-\frac{1}{2}}\right]^{\rho-\frac{p+1}{2}}
$$




$$
=E\left[\left|X_{1}\right|\right]^{\rho-\frac{p+1}{2}} E\left[\mid X_{2}\right]^{-\rho+\frac{p+1}{2}} E\left[X_{3} \mid\right]^{-\rho+\frac{p+1}{2}} \text { due to independence. }
$$

Therefore

$$
M_{g_{1 / 23}}(\rho)=M_{f_{1}}(\rho) M_{f_{2}}(p+1-\rho) M_{f_{3}}(p+1-\rho)
$$

whenever the M-transforms exist. This (4.3) can also be verified by taking the M-transform in (4.2). Take M-transform with respect to $U_{1 / 23}$ by integrating over $f_{1}$ first. Then integrate over $V_{1}$ and $V_{2}$ in $f_{2}$ and $f_{3}$ respectively to see the result in (4.3). Let

$$
U_{1 / 23}=X_{1}^{\frac{1}{2}}\left(X_{3}^{-\frac{1}{2}} X_{2}^{-1} X_{3}^{-\frac{1}{2}}\right) X_{1}^{\frac{1}{2}}=X_{1}^{\frac{1}{2}} U_{32}^{-1} X_{1}^{\frac{1}{2}} .
$$

But the density of $U_{32}$, denoted by $g_{32}$, is the following:

$$
\begin{aligned}
& g_{32}\left(U_{32}\right)=\int_{V_{1}}\left|V_{1}\right|^{-\frac{p+1}{2}} f_{2}\left(V_{1}^{-\frac{1}{2}} U_{32} V_{1}^{-\frac{1}{2}}\right) f_{3}\left(V_{1}\right) \mathrm{d} V_{1}, \\
& g_{32}\left(U_{32}\right)=\int_{V_{1}}\left|V_{1}\right|^{-\frac{p+1}{2}} f_{2}\left(V_{1}\right) f_{3}\left(V_{1}^{-\frac{1}{2}} U_{32} V_{1}^{-\frac{1}{2}}\right) \mathrm{d} V_{1} .
\end{aligned}
$$

Let $X_{1}=V_{2} \Rightarrow U_{32}=V_{2}^{\frac{1}{2}} U_{1 / 23}^{-1} V_{2}^{\frac{1}{2}}$ and the density of $U_{1 / 23}$ is the following:

$$
g_{1 / 23}\left(U_{1 / 23}\right)=\int_{V_{2}}\left|V_{2}\right|^{\frac{p+1}{2}}\left|U_{1 / 23}\right|^{-(p+1)} f_{1}\left(V_{2}\right) g_{32}\left(V_{2}^{\frac{1}{2}} U_{1 / 23}^{-1} V_{2}^{\frac{1}{2}}\right) \mathrm{d} V_{2} \text {. }
$$

Substituting (4.4) and (4.5) for $g_{32}$ in (iii) we have the following:

$$
\begin{aligned}
g_{1 / 23}\left(U_{1 / 23}\right) & =\int_{V_{1}} \int_{V_{2}}\left|V_{1}\right|^{-\frac{p+1}{2}}\left|V_{2}\right|^{\frac{p+1}{2}}\left|U_{1 / 23}\right|^{-(p+1)} f_{1}\left(V_{2}\right) f_{3}\left(V_{1}\right) \\
& \times f_{2}\left(V_{1}^{-\frac{1}{2}} V_{2}^{\frac{1}{2}} U_{1 / 23}^{-1} V_{2}^{\frac{1}{2}} V_{1}^{-\frac{1}{2}}\right) \mathrm{d} V_{1} \wedge \mathrm{d} V_{2}, \\
g_{1 / 23}\left(U_{1 / 23}\right) & =\int_{V_{1}} \int_{V_{2}}\left|V_{1}\right|^{-\frac{p+1}{2}}\left|V_{2}\right|^{\frac{p+1}{2}}\left|U_{1 / 23}\right|^{-(p+1)} f_{1}\left(V_{2}\right) f_{2}\left(V_{1}\right) \\
& \times f_{3}\left(V_{1}^{-\frac{1}{2}} V_{2}^{\frac{1}{2}} U_{1 / 23}^{-1} V_{2}^{\frac{1}{2}} V_{1}^{-\frac{1}{2}}\right) \mathrm{d} V_{1} \wedge \mathrm{d} V_{2} .
\end{aligned}
$$

It can be easily seen that the M-transforms in the M-convolutions of a ratio, namely (4.6) and (4.7), give (4.3). For example, consider (4.6). Take the Mtransform with respect to $U_{1 / 23}$ in $f_{2}$ of (4.6) first. Then integrals over $V_{1}$ and $V_{2}$ give the result. If we take

$$
U_{1 / 23}=U_{32}^{-\frac{1}{2}} X_{1} U_{32}^{-\frac{1}{2}}, U_{32}=V_{2},
$$

then we obtain the following integral representations for $g_{1 / 23}$ : 


$$
\begin{aligned}
g_{1 / 23}\left(U_{1 / 23}\right) & =\int_{V_{1}} \int_{V_{2}}\left|V_{1}\right|^{-\frac{p+1}{2}}\left|V_{2}\right|^{-\frac{p+1}{2}} f_{1}\left(V_{2}^{\frac{1}{2}} U_{1 / 23} V_{2}^{\frac{1}{2}}\right) \\
& \times f_{2}\left(V_{1}^{-\frac{1}{2}} V_{2} V_{1}^{-\frac{1}{2}}\right) f_{3}\left(V_{1}\right) \mathrm{d} V_{1} \wedge \mathrm{d} V_{2}, \\
g_{1 / 23}\left(U_{1 / 23}\right) & =\int_{V_{1}} \int_{V_{2}}\left|V_{1}\right|^{-\frac{p+1}{2}}\left|V_{2}\right|^{\frac{p+1}{2}} f_{1}\left(V_{2}^{\frac{1}{2}} U_{1 / 23} V_{2}^{\frac{1}{2}}\right) \\
& \times f_{2}\left(V_{1}\right) f_{3}\left(V_{1}^{-\frac{1}{2}} V_{2} V_{1}^{-\frac{1}{2}}\right) \mathrm{d} V_{1} \wedge \mathrm{d} V_{2} .
\end{aligned}
$$

It is not difficult to see that the M-transforms in (4.8) and (4.9) give (4.3). For example, in (4.8) take the M-transform with respect to $U_{1 / 23}$ in $f_{1}$ first to get $M_{f_{1}}(\rho)$, then the integral over $V_{2}$ in $f_{2}$ gives $M_{f_{2}}(p+1-\rho)$ and then the integral over $V_{1}$ in $f_{3}$ gives $M_{f_{3}}(p+1-\rho)$.

We can also take

$$
\begin{gathered}
U_{1 / 23}=X_{3}^{-\frac{1}{2}} X_{1}^{\frac{1}{2}} X_{2}^{-1} X_{1}^{\frac{1}{2}} X_{3}^{-\frac{1}{2}} \\
V_{1}=X_{3}, V_{2}=X_{1} \Rightarrow X_{2}=V_{2}^{\frac{1}{2}} V_{1}^{-\frac{1}{2}} U_{1 / 23}^{-1} V_{1}^{-\frac{1}{2}} V_{2}^{\frac{1}{2}} .
\end{gathered}
$$

We can also take

$$
U_{1 / 23}=X_{2}^{-\frac{1}{2}} X_{1}^{\frac{1}{2}} X_{3}^{-1} X_{1}^{\frac{1}{2}} X_{2}^{-\frac{1}{2}}, V_{1}=X_{2}, V_{2}=X_{1}
$$

These produce the following integral representations:

$$
\begin{aligned}
g_{1 / 23}\left(U_{1 / 23}\right) & =\int_{V_{1}} \int_{V_{2}}\left|V_{1}\right|^{-\frac{p+1}{2}}\left|V_{2}\right|^{\frac{p+1}{2}}\left|U_{1 / 23}\right|^{-(p+1)} f_{1}\left(V_{1}\right) \\
& \times f_{2}\left(V_{2}^{\frac{1}{2}} V_{1}^{-\frac{1}{2}} U_{1 / 23}^{-1} V_{1}^{-\frac{1}{2}} V_{2}^{\frac{1}{2}}\right) f_{3}\left(V_{1}\right) \mathrm{d} V_{1} \wedge \mathrm{d} V_{2}, \\
g_{1 / 23}\left(U_{1 / 23}\right) & =\int_{V_{1}} \int_{V_{2}}\left|V_{1}\right|^{-\frac{p+1}{2}}\left|V_{2}\right|^{\frac{p+1}{2}}\left|U_{1 / 23}\right|^{-(p+1)} f_{2}\left(V_{1}\right) \\
& \times f_{3}\left(V_{2}^{\frac{1}{2}} V_{1}^{-\frac{1}{2}} U_{1 / 23}^{-1} V_{1}^{-\frac{1}{2}} V_{2}^{\frac{1}{2}}\right) \mathrm{d} V_{1} \wedge \mathrm{d} V_{2} .
\end{aligned}
$$

\subsection{Fractional integrals}

In (4.6) let $f_{2}\left(X_{2}\right)$ have a real matrix-variate type-1 beta density with the parameters $(\gamma, \alpha)$ or with the density

$$
f_{2}\left(X_{2}\right)=\frac{\Gamma_{p}(\gamma+\alpha)}{\Gamma_{p}(\gamma) \Gamma_{p}(\alpha)}\left|X_{2}\right|^{\gamma-\frac{p+1}{2}}\left|I-X_{2}\right|^{\alpha-=\frac{p+1}{2}},
$$

$0<X_{2}<I, \gamma>\frac{p-1}{2}, \alpha>\frac{p-1}{2}$. Then 


$$
\begin{aligned}
& \left|V_{1}\right|^{-\frac{p+1}{2}}\left|V_{2}\right|^{\frac{p+1}{2}}\left|U_{1 / 23}\right|^{-(p+1)} f_{2}\left(V_{1}^{-\frac{1}{2}} V_{2}^{\frac{1}{2}} U_{1 / 23}^{-1} V_{2}^{\frac{1}{2}} V_{1}^{-\frac{1}{2}}\right) \\
& =\frac{\Gamma_{p}(\gamma+\alpha)}{\Gamma_{p}(\gamma)} \frac{\left|U_{1 / 23}\right|^{-\alpha-\gamma}}{\Gamma_{p}(\alpha)}\left|V_{1}^{-1}\right|^{\gamma}\left|V_{2}\right|^{\gamma}\left|U_{1 / 23}-V_{2}^{\frac{1}{2}} V_{1}^{-1} V_{2}^{\frac{1}{2}}\right|^{\alpha-\frac{p+1}{2}} .
\end{aligned}
$$

Then $g_{1 / 23}$ in (4.6) becomes the following for $\Re(\alpha)>\frac{p-1}{2}, \Re(\gamma)>\frac{p-1}{2}$ :

$$
\begin{aligned}
g_{1 / 23}\left(U_{1 / 23}\right) & =\frac{\Gamma_{p}(\gamma+\alpha)}{\Gamma_{p}(\gamma)} \frac{\left|U_{1 / 23}\right|^{-\alpha-\gamma}}{\Gamma_{p}(\alpha)} \iint_{V_{2}^{\frac{1}{2}} V_{1}^{-1} V_{2}^{\frac{1}{2}}<U_{1 / 23}}\left|V_{1}^{-1}\right|^{\gamma}\left|V_{2}\right|^{\gamma} \\
& \times\left|U_{1 / 23}-V_{2}^{\frac{1}{2}} V_{1}^{-1} V_{2}^{\frac{1}{2}}\right|^{\alpha-\frac{p+1}{2}} f_{3}\left(V_{1}\right) f_{2}\left(V_{2}\right) \mathrm{d} V_{1} \wedge \mathrm{d} V_{2} \\
& =\frac{\Gamma_{p}(\gamma+\alpha)}{\Gamma_{p}(\gamma)} K_{1, \gamma}^{-\alpha}\left(f_{3}, f_{2}\right)
\end{aligned}
$$

where $K_{1, \gamma}^{-\alpha}\left(f_{3}, f_{2}\right)$ has the structure of an extended Erdélyi-Kober fractional integral of the first kind of order $\alpha$ and parameter $\gamma$ with two arbitrary functions $f_{3}$ and $f_{2}$. If $f_{3}$ and $V_{1}$ are absent then it is a real matrix-variate fractional integral of the first kind of order $\alpha$ and parameter $\gamma$, see [7]. Hence (4.13) is a type of extension of fractional integral of the first kind.

If $f_{2}$ in (4.10) has a real matrix-variate type- 1 beta density with the parameters $(\gamma, \alpha)$ or with the density given in $(i v)$, then consider

$$
\begin{aligned}
& \left|V_{1}\right|^{-\frac{p+1}{2}}\left|V_{2}\right|^{\frac{p+1}{2}}\left|U_{1 / 23}\right|^{-(p+1)} f_{2}\left(V_{2}^{\frac{1}{2}} V_{1}^{-\frac{1}{2}} U_{1 / 23}^{-1} V_{1}^{-\frac{1}{2}} V_{2}^{\frac{1}{2}}\right) \\
& =\frac{\Gamma_{p}(\gamma+\alpha)}{\Gamma_{p}(\gamma)} \frac{\left|U_{1 / 23}\right|^{-\gamma-\alpha}}{\Gamma_{p}(\alpha)}\left|V_{1}^{-1}\right|^{\gamma}\left|V_{2}\right|^{\gamma}\left|U_{1 / 23}-V_{1}^{-\frac{1}{2}} V_{2} V_{1}^{-\frac{1}{2}}\right|^{\alpha-\frac{p+1}{2}} .
\end{aligned}
$$

Then (4.10) reduces to the following form for $\Re(\gamma)>\frac{p-1}{2}, \Re(\alpha)>\frac{p-1}{2}$ :

$$
\begin{aligned}
g_{1 / 23}\left(U_{1 / 23}\right) & =\frac{\Gamma_{p}(\gamma+\alpha)}{\Gamma_{p}(\gamma)} \frac{\left|U_{1 / 23}\right|^{-\gamma-\alpha}}{\Gamma_{p}(\alpha)} \iint_{V_{1}^{-\frac{1}{2}} V_{2} V_{1}^{-\frac{1}{2}}<U_{1 / 23}}\left|V_{1}^{-1}\right|^{\gamma}\left|V_{2}\right|^{\gamma} \\
& \times\left|U_{1 / 23}-V_{1}^{-\frac{1}{2}} V_{2} V_{1}^{-\frac{1}{2}}\right|^{\alpha-\frac{p+1}{2}} f_{3}\left(V_{1}\right) f_{2}\left(V_{2}\right) \mathrm{d} V_{1} \wedge \mathrm{d} V_{2} \\
& =\frac{\Gamma_{p}(\gamma+\alpha)}{\Gamma_{p}(\gamma)} \frac{\left|U_{1 / 23}\right|^{-\gamma-\alpha}}{\Gamma_{p}(\alpha)} K_{1, \gamma}^{-\alpha}\left(f_{3}, f_{2}\right)
\end{aligned}
$$

where $K_{1, \gamma}^{-\alpha}\left(f_{3}, f_{2}\right)$ corresponds to a real matrix-variate fractional integral of the first kind of order $\alpha$ and parameter $\gamma$ involving two arbitrary functions $f_{3}$ and $f_{2}$. If $f_{3}$ and $V_{1}$ are absent then it is the Erdélyi-Kober fractional integral 
of the first kind of order $\alpha$ and parameter $\gamma$ in the real matrix-variate case, see $[7]$.

Other symmetric ratio forms involving three matrices where two are in the denominator and one is in the numerator will give other integral representations, some of which will coincide with the structural representations discussed above and others will be different.

\section{M-convolutions of products and ratios in the complex domain}

In the complex domain, one can obtain results parallel to all the results in the real matrix-variate case plus more. Since the manuscript is getting too large, we will stop here after indicating how to obtain results in the complex case. Basic Jacobians that will be needed are available in [5] and the essential results on Jacobians will be given here as a lemma. Observe that when a $p \times p$ matrix $\tilde{X}$ is Hermitian we have $\tilde{X}=\tilde{X}^{*}$, where $\tilde{X}^{*}$ indicates the conjugate transpose of $\tilde{X}$. In general, a $p \times p$ matrix $\tilde{X}$ in the complex domain can be written as $\tilde{X}=X_{1}+i X_{2}, i=\sqrt{(-1)}, X_{1}, X_{2}$ real. If $\tilde{X}=\tilde{X}^{*}$ then $X_{1}=X_{1}^{\prime}$ (symmetric) and $X_{2}=-X_{2}^{\prime}$ (skew symmetric). Then $X_{1}$ has $p(p+1) / 2$ real scalar variables and $X_{2}$ has $p(p-1) / 2$ real scalar variables.

Lemma 5.1. Let $\tilde{X}=\tilde{X}^{*}$ be a $p \times p$ Hermitian matrix and $A$ a $p \times p$ constant nonsingular matrix. Then

$$
\tilde{Y}=A \tilde{X} A^{*} \Rightarrow \mathrm{d} \tilde{Y}=|\operatorname{det}(A)|^{2 p} \mathrm{~d} \tilde{X}=\left|\operatorname{det}\left(A A^{*}\right)\right|^{p} \mathrm{~d} \tilde{X}
$$

When $\tilde{X}$ is $p \times p$ nonsingular then

$$
\tilde{Y}=\tilde{X}^{-1} \Rightarrow \mathrm{d} \tilde{Y}=\left\{\begin{array}{l}
\left|\operatorname{det}\left(\tilde{X} \tilde{X}^{*}\right)\right|^{-2 p} \mathrm{~d} \tilde{X} \text { for a general } \tilde{X} \\
\left|\operatorname{det}\left(\tilde{X} \tilde{X}^{*}\right)\right|^{-p} \text { for } \tilde{X}=\tilde{X}^{*} \text { or } \tilde{X}=-\tilde{X}^{*},
\end{array}\right.
$$

where $\mathrm{d} \tilde{X}=\mathrm{d} X_{1} \wedge \mathrm{d} X_{2}, \tilde{X}=X_{1}+i X_{2}, i=\sqrt{(-1)}, X_{1}, X_{2}$ real.

With the help of Lemma 5.1 we will illustrate two results and then stop this section. Let $\tilde{X}_{j}, j=1,2,3$ be $p \times p$ Hermitian positive definite matrix random variables, independently distributed with the densities $\tilde{f}\left(\tilde{X}_{j}\right), j=1,2,3$, respectively, $\tilde{f}\left(\tilde{X}_{j}\right) \geq 0$ for all $\tilde{X}_{j}, \int_{\tilde{X}_{j}} \tilde{f}_{j}\left(\tilde{X}_{j}\right) \mathrm{d} \tilde{X}_{j}=1, j=1,2,3$, where $\tilde{f}_{j}$ is a real-valued scalar function of $\tilde{X}_{j}$. Due to independence, the joint density of $\tilde{X}_{j}, j=1,2,3$ is $\tilde{f}_{1}\left(\tilde{X}_{1}\right) \tilde{f}_{2}\left(\tilde{X}_{2}\right) \tilde{f}_{3}\left(\tilde{X}_{3}\right)$. Consider the symmetric product

$$
\tilde{U}_{123}=\tilde{X}_{1}^{\frac{1}{2}} \tilde{X}_{2}^{\frac{1}{2}} \tilde{X}_{3} \tilde{X}_{2}^{\frac{1}{2}} \tilde{X}_{1}^{\frac{1}{2}} \text {, with } \tilde{V}_{1}=\tilde{X}_{1}, \tilde{V}_{2}=\tilde{X}_{2}
$$




$$
\begin{gathered}
\Rightarrow \tilde{X}_{3}=\tilde{V}_{2}^{-\frac{1}{2}} \tilde{V}_{1}^{-\frac{1}{2}} \tilde{U}_{123} \tilde{V}_{1}^{-\frac{1}{2}} \tilde{V}_{2}^{-\frac{1}{2}} \text { and } \\
\mathrm{d} \tilde{X}_{1} \wedge \mathrm{d} \tilde{X}_{2} \wedge \mathrm{d} \tilde{X}_{3}=\left|\operatorname{det}\left(\tilde{V}_{1}\right)\right|^{-p}\left|\operatorname{det}\left(\tilde{V}_{2}\right)\right|^{-p} \mathrm{~d} \tilde{V}_{1} \wedge \mathrm{d} \tilde{V}_{2} \wedge \mathrm{d} \tilde{U}_{123} .
\end{gathered}
$$

The Jacobian in $(i)$ is obtained by the repeated application of (5.1) of Lemma 5.1. Then the density of $\tilde{U}_{123}$, denoted by $\tilde{g}_{123}\left(\tilde{U}_{123}\right)$, is given by the following:

$$
\begin{aligned}
\tilde{g}_{123}\left(\tilde{U}_{123}\right) & =\int_{\tilde{V}_{1}>O} \int_{\tilde{V}_{2}>O}\left|\operatorname{det}\left(\tilde{V}_{1}\right)\right|^{-p}\left|\operatorname{det}\left(\tilde{V}_{2}\right)\right|^{-p} \tilde{f}_{1}\left(\tilde{V}_{1}\right) \tilde{f}\left(\tilde{V}_{2}\right) \\
& \times \tilde{f}_{3}\left(\tilde{V}_{2}^{-\frac{1}{2}} \tilde{V}_{1}^{-\frac{1}{2}} \tilde{U}_{123} \tilde{V}_{1}^{-\frac{1}{2}} \tilde{V}_{2}^{-\frac{1}{2}}\right) \mathrm{d} \tilde{V}_{1} \wedge \mathrm{d} \tilde{V}_{2} .
\end{aligned}
$$

When $\tilde{f}_{j}, j=1,2,3$ are general functions or densities, then $\tilde{g}_{123}$ is called the M-convolution of a product involving three functions in the complex domain, and when $\tilde{f}_{j}, j=1,2,3$ are densities then $\tilde{g}_{123}$ is also the unique statistical density of a symmetric product in the complex domain. The M-convolution of a product property is that if we take the M-transform of $\tilde{g}_{123}$ then we get the product of the M-transforms of $\tilde{f}_{j}, j=1,2,3$. The M-transform of a real-valued scalar function $\tilde{f}(\tilde{X})$ of a $p \times p$ Hermitian positive definite matrix $\tilde{X}$ is defined and denoted as follows:

$$
\tilde{M}_{\tilde{f}}(\rho)=\int_{\tilde{X}>O}|\operatorname{det}(\tilde{X})|^{\rho-p} \tilde{f}(\tilde{X}) \mathrm{d} \tilde{X}, \quad \Re(\rho)>p-1,
$$

whenever the integral is convergent. Let us consider the M-transform of $\tilde{g}_{123}$, with parameter $\rho$. Then

$$
\tilde{M}_{\tilde{g}_{123}}(\rho)=\int_{\tilde{U}_{123}>O}\left|\operatorname{det}\left(\tilde{U}_{123}\right)\right|^{\rho-p} \tilde{g}_{123}\left(\tilde{U}_{123}\right) \mathrm{d} \tilde{U}_{123} .
$$

Take the integral in $\tilde{f}_{3}$ first under the condition of interchange of integrals,

$$
\begin{aligned}
& \int_{\tilde{U}_{123}}\left|\operatorname{det}\left(\tilde{U}_{123}\right)\right|^{\rho-p} \tilde{f}_{3}\left(\tilde{V}_{2}^{-\frac{1}{2}} \tilde{V}_{1}^{-\frac{1}{2}} \tilde{U}_{123} \tilde{V}_{1}^{-\frac{1}{2}} \tilde{V}_{2}^{-\frac{1}{2}}\right) \mathrm{d} \tilde{U}_{123} \\
& =\left|\operatorname{det}\left(\tilde{V}_{1}\right)\right|^{\rho}\left|\operatorname{det}\left(\tilde{V}_{2}\right)\right|^{\rho} \int_{\tilde{T}>O}|\operatorname{det}(\tilde{T})|^{\rho-p} \mathrm{~d} \tilde{T}, \\
& \tilde{T}=\tilde{V}_{2}^{-\frac{1}{2}} \tilde{V}_{1}^{-\frac{1}{2}} \tilde{U}_{123} \tilde{V}_{1}^{-\frac{1}{2}} \tilde{V}_{2}^{-\frac{1}{2}}=\left|\operatorname{det}\left(\tilde{V}_{1}\right)\right|^{\rho}\left|\operatorname{det}\left(\tilde{V}_{2}\right)\right|^{\rho} \tilde{M}_{\tilde{f}_{3}}(\rho)
\end{aligned}
$$

whenever the M-transform exists. Now, integration over $\tilde{V}_{1}$ and $\tilde{V}_{2}$ give $\tilde{M}_{\tilde{f}_{1}}(\rho) \tilde{M}_{\tilde{f}_{2}}(\rho)$. In other words,

$$
\tilde{M}_{\tilde{g}_{123}}(\rho)=\tilde{M}_{\tilde{f}_{1}}(\rho) \tilde{M}_{\tilde{f}_{2}}(\rho) \tilde{M}_{\tilde{f}_{3}}(\rho)
$$

whenever the M-transforms exist. 
Now, let us consider an application of (5.3). Let $\tilde{f}_{3}\left(\tilde{X}_{3}\right)$ be a matrix-variate type- 1 beta density in the complex domain with the parameters $(\gamma+p, \alpha)$ or with the density

$$
\tilde{f}_{3}\left(\tilde{X}_{3}\right)=\frac{\tilde{\Gamma}_{p}(\gamma+p+\alpha)}{\tilde{\Gamma}_{p}(\gamma+p) \tilde{\Gamma}_{p}(\alpha)}\left|\operatorname{det}\left(\tilde{X}_{3}\right)\right|^{\gamma}\left|\operatorname{det}\left(I-\tilde{X}_{3}\right)\right|^{\alpha-p},
$$

for $\tilde{X}_{3}>0, I-\tilde{X}_{3}>0, \gamma>-1, \alpha>p-1$ and if the parameters are complex, then $\Re(\gamma)>-1, \Re(\alpha)>p-1$, where for example $\tilde{\Gamma}_{p}(\alpha)$ means the complex matrix-variate gamma function given by

$$
\tilde{\Gamma}_{p}(\alpha)=\pi^{\frac{p(p-1)}{2}} \Gamma(\alpha) \Gamma(\alpha-1) \ldots \Gamma(\alpha-p+1), \Re(\alpha)>p-1 .
$$

The derivation of $\tilde{\Gamma}_{p}(\alpha)$ is available from [5]. Consider

$$
\begin{aligned}
& \left|\operatorname{det}\left(\tilde{V}_{1}\right)\right|^{-\rho}\left|\operatorname{det}\left(\tilde{V}_{2}\right)\right|^{-\rho} \tilde{f}_{3}\left(\tilde{V}_{2}^{-\frac{1}{2}} \tilde{V}_{1}^{-\frac{1}{2}} \tilde{U}_{123} \tilde{V}_{1}^{-\frac{1}{2}} \tilde{V}_{2}^{-\frac{1}{2}}\right) \\
& =\frac{\tilde{\Gamma}_{p}(\gamma+p+\alpha)}{\tilde{\Gamma}_{p}(\gamma+p) \tilde{\Gamma}_{p}(\alpha)}\left|\operatorname{det}\left(\tilde{V}_{1}\right)\right|^{-\rho}\left|\operatorname{det}\left(\tilde{V}_{2}\right)\right|^{-\rho}\left|\operatorname{det}\left(\tilde{V}_{2}^{-\frac{1}{2}} \tilde{V}_{1}^{-\frac{1}{2}} \tilde{U}_{123} \tilde{V}_{1}^{-\frac{1}{2}} \tilde{V}_{2}^{-\frac{1}{2}}\right)\right|^{\gamma} \\
& \times\left|\operatorname{det}\left(I-\tilde{V}_{2}^{-\frac{1}{2}} \tilde{V}_{1}^{-\frac{1}{2}} \tilde{U}_{123} \tilde{V}_{1}^{-\frac{1}{2}} \tilde{V}_{2}^{-\frac{1}{2}}\right)\right|^{\alpha-p} \\
& \left.=\frac{\tilde{\Gamma}_{p}(\gamma+p+\alpha)}{\tilde{\Gamma}_{p}(\gamma+p)} \frac{\left|\operatorname{det}\left(\tilde{U}_{123}\right)\right|^{\gamma}}{\tilde{\Gamma}_{p}(\alpha)} \mid \operatorname{det} \tilde{V}_{1}\right)\left.\right|^{-\alpha-\gamma}\left|\operatorname{det}\left(\tilde{V}_{2}\right)\right|^{-\alpha-\gamma} \\
& \times\left|\operatorname{det}\left(\tilde{V}_{1}^{\frac{1}{2}} \tilde{V}_{2} \tilde{V}_{1}^{\frac{1}{2}}-\tilde{U}_{123}\right)\right|^{\alpha-p} .
\end{aligned}
$$

Now, (5.3) becomes the following for $\Re(\gamma)>p-1, \Re(\alpha)>p-1$ :

$$
\begin{aligned}
\tilde{g}_{123}\left(\tilde{U}_{123}\right) & =\frac{\tilde{\Gamma}_{p}(\gamma+p+\alpha)}{\tilde{\Gamma}_{p}(\gamma+p)} \frac{\left|\operatorname{det}\left(\tilde{U}_{123}\right)\right|^{\gamma}}{\tilde{\Gamma}_{p}(\alpha)} \iint_{\tilde{V}_{1}^{\frac{1}{2}} \tilde{V}_{2} \tilde{V}_{1}^{\frac{1}{2}}>\tilde{U}_{123}}\left|\operatorname{det}\left(\tilde{V}_{1}\right)\right|^{-\alpha-\gamma} \\
& \times\left|\operatorname{det}\left(\tilde{V}_{2}\right)\right|^{-\alpha-\gamma} \tilde{f}_{1}\left(\tilde{V}_{1}\right) \tilde{f}_{2}\left(\tilde{V}_{2}\right) \mathrm{d} \tilde{V}_{1} \wedge \mathrm{d} \tilde{V}_{2} \\
& =\frac{\tilde{\Gamma}_{p}(\gamma+p+\alpha)}{\tilde{\Gamma}_{p}(\gamma+p)} \tilde{K}_{2, \gamma}^{-\alpha}\left(\tilde{f}_{1}, \tilde{f}_{2}\right)
\end{aligned}
$$

for $\Re(\gamma)>-1, \Re(\alpha)>p-1$ where $\tilde{K}_{2, \gamma}^{-\alpha}\left(\tilde{f}_{1}, \tilde{f}_{2}\right)$ can be called an extension of Erdélyi-Kober fractional integral of the second kind of order $\alpha$ and parameter $\gamma$ in the complex domain for two arbitrary functions $\tilde{f}_{1}$ and $\tilde{f}_{2}$. For matrixvariate fractional integrals in the complex domain, see [6]. When $\tilde{f}_{1}$ and $\tilde{V}_{1}$ or $\tilde{f}_{2}$ and $\tilde{V}_{2}$ are absent then (5.8) is the complex matrix-variate fractional integral of the second kind of order $\alpha$ and parameter $\gamma$ as described in [6]. Observe that when $\tilde{f}_{j}, j=1,2,3$ are densities then the Erdélyi-Kober fractional integral 
$\tilde{K}_{2, \gamma}^{-\alpha}\left(\tilde{f}_{1}, \tilde{f}_{2}\right)$ is a constant multiple of a statistical density $\tilde{g}_{123}$, otherwise it is a constant multiple of a M-convolution in the complex case involving three functions.

We will consider an illustration of a symmetric ratio also. Consider a ratio where two complex matrices are in the numerator and one complex matrix is in the denominator. Let

$$
\begin{aligned}
\tilde{U}_{12 / 3} & =\tilde{X}_{1}^{\frac{1}{2}} \tilde{X}_{2}^{\frac{1}{2}} \tilde{X}_{3}^{-1} \tilde{X}_{2}^{\frac{1}{2}} \tilde{X}_{1}^{\frac{1}{2}} \text { with } \tilde{X}_{1}=\tilde{V}_{1}, \tilde{X}_{2}=\tilde{V}_{2} \\
& \Rightarrow \tilde{X}_{3}=\tilde{V}_{2}^{\frac{1}{2}} \tilde{V}_{1}^{\frac{1}{2}} \tilde{U}_{12 / 3}^{-1} \tilde{V}_{1}^{\frac{1}{2}} \tilde{V}_{2}^{\frac{1}{2}} \Rightarrow \\
& \mathrm{d} \tilde{X}_{1} \wedge \mathrm{d} \tilde{X}_{2} \wedge \mathrm{d} \tilde{X}_{3} \\
& =\left|\operatorname{det}\left(\tilde{V}_{1}\right)\right|^{p}\left|\operatorname{det}\left(\tilde{V}_{2}\right)\right|^{p}\left|\operatorname{det}\left(\tilde{U}_{12 / 3}\right)\right|^{-2 p} \\
& \mathrm{~d} \tilde{V}_{1} \wedge \mathrm{d} \tilde{V}_{2} \wedge \mathrm{d} \tilde{U}_{12 / 3} .
\end{aligned}
$$

Then the density of $\tilde{U}_{12 / 3}$, denoted by $\tilde{g}_{12 / 3}\left(\tilde{U}_{12 / 3}\right)$ is the following:

$$
\begin{aligned}
\tilde{g}_{12 / 3}\left(\tilde{U}_{12 / 3}\right) & =\int_{\tilde{V}_{1}} \int_{\tilde{V}_{2}}\left|\operatorname{det}\left(\tilde{V}_{1}\right)\right|^{p}\left|\operatorname{det}\left(\tilde{V}_{2}\right)\right|^{p}\left|\operatorname{det}\left(\tilde{U}_{12 / 3}\right)\right|^{-2 p} \tilde{f}_{1}\left(\tilde{V}_{1}\right) \tilde{f}_{2}\left(\tilde{V}_{2}\right) \\
& \times \tilde{f}_{3}\left(\tilde{V}_{2}^{\frac{1}{2}} \tilde{V}_{1}^{\frac{1}{2}} \tilde{U}_{12 / 3}^{-1} \tilde{V}_{1}^{\frac{1}{2}} \tilde{V}_{2}^{\frac{1}{2}}\right) \mathrm{d} \tilde{V}_{1} \wedge \mathrm{d} \tilde{V}_{2} .
\end{aligned}
$$

When $\tilde{f}_{j} \cdot j=1,2,3$ are densities then $\tilde{g}_{12 / 3}$ is a density and when $\tilde{f}_{j} \cdot j=1,2,3$ are densities or not then $\tilde{g}_{12 / 3}$ is the M-convolution of a ratio involving three functions $\tilde{f}_{j}, j=1,2,3$ in the complex case. Proceeding as before, we can see that the M-transform of $\tilde{g}_{12 / 3}$ will be of the following form:

$$
\tilde{M}_{\tilde{g}_{12 / 3}}(\rho)=\tilde{M}_{\tilde{f}_{1}}(\rho) \tilde{M}_{\tilde{f}_{2}}(\rho) \tilde{M}_{\tilde{f}_{3}}(2 p-\rho)
$$

whenever the M-transforms exist. This is the M-convolution of a ratio property in the complex case.

We will consider one application. Let $\tilde{f}_{3}$ be a complex matrix-variate type-1 beta density with the parameters $(\gamma, \alpha)$ or with the density

$$
\tilde{f}_{3}\left(\tilde{X}_{3}\right)=\frac{\tilde{\Gamma}_{p}(\gamma+\alpha)}{\tilde{\Gamma}_{p}(\gamma) \tilde{\Gamma}_{p}(\alpha)}\left|\operatorname{det}\left(\tilde{X}_{3}\right)\right|^{\gamma-p}\left|\operatorname{det}\left(I-\tilde{X}_{3}\right)\right|^{\alpha-p}
$$

for $\tilde{X}_{3}>O, I-\tilde{X}_{3}>O, \Re(\gamma)>p-1, \Re(\alpha)>p-1$. Then

$$
\begin{aligned}
& \left|\operatorname{det}\left(\tilde{V}_{1}\right)\right|^{p}\left|\operatorname{det}\left(\tilde{V}_{2}\right)\right|^{p}\left|\operatorname{det}\left(\tilde{U}_{12 / 3}\right)\right|^{-2 p} \tilde{f}_{3}\left(\tilde{V}_{2}^{\frac{1}{2}} \tilde{V}_{1}^{\frac{1}{2}} \tilde{U}_{12 / 3}^{-1} \tilde{V}_{1}^{\frac{1}{2}} \tilde{V}_{2}^{\frac{1}{2}}\right) \\
& =\left|\operatorname{det}\left(\tilde{U}_{12 / 3}\right)\right|^{-\alpha-\gamma}\left|\operatorname{det}\left(\tilde{V}_{1}\right)\right|^{\gamma}\left|\operatorname{det}\left(\tilde{V}_{2}\right)\right|^{\gamma}\left|\operatorname{det}\left(\tilde{U}_{12 / 3}-\tilde{V}_{1}^{\frac{1}{2}} \tilde{V}_{2} \tilde{V}_{1}^{\frac{1}{2}}\right)\right|^{\alpha-p} .
\end{aligned}
$$


Then (5.9) becomes the following:

$$
\begin{aligned}
\tilde{g}_{12 / 3}\left(\tilde{U}_{12 / 3}\right) & =\frac{\tilde{\Gamma}_{p}(\gamma+\alpha)}{\tilde{\Gamma}_{p}(\gamma)} \frac{\left|\operatorname{det}\left(\tilde{U}_{12 / 3}\right)\right|^{-\alpha-\gamma}}{\tilde{\Gamma}_{p}(\alpha)} \iint_{\tilde{V}_{1}^{\frac{1}{2}} \tilde{V}_{2} \tilde{V}_{1}^{\frac{1}{2}}<\tilde{U}_{12 / 3}}\left|\operatorname{det}\left(\tilde{V}_{1}\right)\right|^{\gamma} \\
& \times\left|\operatorname{det}\left(\tilde{V}_{2}\right)\right|^{\gamma} \tilde{f}_{1}\left(\tilde{V}_{1}\right) \tilde{f}_{2}\left(\tilde{V}_{2}\right) \mathrm{d} \tilde{V}_{1} \wedge \mathrm{d} \tilde{V}_{2} \\
& =\frac{\tilde{\Gamma}_{p}(\gamma+\alpha)}{\tilde{\Gamma}_{p}(\gamma)} \tilde{K}_{1, \gamma}^{-\alpha}\left(\tilde{f}_{1}, \tilde{f}_{2}\right)
\end{aligned}
$$

for $\Re(\alpha)>p-1, \Re(\gamma)>p-1$, where $\tilde{K}_{1, \gamma}^{-\alpha}\left(\tilde{f}_{1}, \tilde{f}_{2}\right)$ is an extended Erdélyi-Kober fractional integral of the first kind in the complex case of order $\alpha$ and parameter $\gamma$ on two arbitrary functions $\tilde{f}_{1}$ and $\tilde{f}_{2}$. For the one arbitrary function case see [6].

Note. This research did not receive any specific grant from funding agencies in the public, commercial, or not-for-profit sectors.

\section{References}

[1] V. Kiryakova and Yu Luchko, Riemann-Liouville and Caputo type multiple Erdélyi-Kober operators, Central European J. of Physics, 11, No 10 (2013), 1314-1336; DOI: 10.2478/s11534-013-0217-1.

[2] E. Krätzel, Integral transformation of Bessel type, In: Generalized Functions \& Operational Calculus (Proc. Conf. Varna, 1975), Bulg. Acad. Sci., Sofia, 1979, 148-165.

[3] F. Mainardi, Yu Luchko and G. Pagnini, The fundamental solutions of the space-time fractional diffusion equation, Fract. Calc. Appl. Anal., 4, No 2 (2001), 153-192.

[4] A.M. Mathai, Some results on functions of matrix argument, Mathematische Nachrichten, 84 (1978), 171-177.

[5] A.M. Mathai, Jacobians of Matrix Transformations and Functions of Matrix Argument, World Scientific Publishing, New York, 1997.

[6] A.M. Mathai, Fractional integral operators in the complex matrix-variate case, Linear Algebra and its Applications, 439 (2013), 2901-2913.

[7] A.M. Mathai, Fractional integral operators involving many matrix variables, Linear Algebra and its Applications, 446 (2014), 196-215. 
[8] A.M. Mathai, Mellin convolution, statistical distributions and fractional calculus, Fract. Calc. Appl. Anal., 21, No 2 (2018), 376-398; DOI: 10.1515/fca-2018-0022.

[9] A.M. Mathai and H.J. Haubold, Modern Problems in Nuclear and Neutrino Astrophysics, Akademie-Verlag, Berlin, 1988. 